\title{
Time fractional heat transfer analysis in nonhomogeneous thick hollow cylinder with internal heat generation and its thermal stresses
}

\author{
${ }^{1}$ Shivcharan Thakare, ${ }^{2} \mathrm{M} S \mathrm{~S}$ Warbhe and ${ }^{3}$ Navneet Kumar \\ ${ }^{1,2}$ Department of Mathematics, Sarvodaya Mahavidyalaya, Sindewahi, Chandrapur, India \\ ${ }^{3}$ Department of Mathematics, S.L.P.M., Mandhal RTM Nagpur University, Nagpur, India \\ Email: navneet19021984kumar@gmail.com
}

Received 6 May 2020, Accepted 2 October 2020

\begin{abstract}
The present article deals with the study of a two dimensional thermoelastic problem of nonhomogeneous thick hollow cylinder within the context of fractional order derivative of order $0<\alpha \leq 2$. In which convection boundary conditions are applied on the curved surface of cylinder with internal heat generation. The material properties other then Poisson's ratio and density are expresses by a simple power law in axial direction. Also lower and upper surface are assumed to be thermally insulated. The affect of inhomogeneity on the both thermal and mechanical behavior is determined. Numerical computations are carried out with the help of Mathematica software for both homogeneous and nonhomogeneous cylinders as well as illustrated graphically in figures.
\end{abstract}

Keywords: Time fractional; nonhomogeneous cylinder; thermal stresses; internal heat generation.

\section{Introduction}

Nonhomogeneous materials are those materials which have different non-uniform material properties. These materials are also known as heterogeneous materials, which contain a mixture of various metallic elements. Due to superior mechanical properties these materials plays significant role in design of future intelligent composites which have great applications in science and engineering. Thermoelastic deformation and stresses in the case of semi-infinite nonhomogeneous solids where rigidity modulus varies with its depth according to a power law function were successfully investigated by Kassir [5]. Thermal stress distribution for a nonhomogeneous plate where shear modulus and thermal expansions coefficient were considered as a function of $z$ under steady state temperature condition was investigated by Hata [9]. One-dimensional transient temperature distribution in a functionally graded composed circular hollow cylinder was analyzed numerically by Awaji and Sivakumar [15]. The 2 D unsteady state thermoelastic problem of an infinite hollow functionally graded material circular cylinder with dependent properties along radial direction using Green's function approach were calculated by Kim and Noda [16]. A new modified integral transform was developed to investigate a mixed boundary value problem which involves combination of Bessel's function as a kernel by Al-Hajri and Kalla [18]. Ootao and Tanigawa [20, 25 and 37] studied various thermoelastic problems for hollow cylinder due to uniform heat supply with nonhomogeneous piecewise power law in Laplace transform domain. Sugano [10, 11 and 12] derived transient thermal stresses in a doubly connected non-homogeneous region where Young's modulus and thermal conductivity presented in radial coordinate power laws. Thermoelastic analysis subjected to partially heating on curved surface of a circular plate was investigated by Deshmukh and Khobragade [19]. Transient thermoelastic solution of functionally graded thick hollow cylinders was obtained analytically by Hosseini and Akhlaghi [24]. In [28], Solution for the temperature and thermal stresses due to circumferential loading in a hollow cylinder using integral transform was determined successfully. In [46], the analysis of thermo elastic stress due to action of thermo mechanical loading of a laminated isotropic materials cylinder done. In [47] Thermoelastic behaviour in a solid cylinder with circumferential crack was studied using theory based on $\mathrm{C}-\mathrm{V}$ heat conduction. In [48], the buckling effect presented for a solid circular plate made of porous material. Temperature distribution and thermal deflection on the 
outer curved surface of a semi-infinite hollow circular cylinder was studied by Kedar and Deshmukh [54].

In year 1822, French mathematician Jean Baptiste Fourier gives a relationship between the heat flow and the temperature gradient for a stationary, homogeneous and isotropic solid with this classical theory of heat conduction begin. After some year Duhamel's relates heat conduction boundary value problems with time-dependent boundary conditions which work as base for mathematical foundation of thermoelasticity. Also it is testify that classical Fourier law and heat conduction equation in parabolic form are no longer accurate in many studies based on theoretical and experimental approach of transport phenomenon in media with internal structure like porous, polymers, dielectrics, semiconductors, amorphous etc. So the physical process exist at the microscopic level should be held into account. Therefore microscopic level is quite essential for different physical situations but this ignores during processing by the classical Fourier law. This encourages for the formulation of nonclassical theories, which implies to replace the parabolic heat conduction equation and the Fourier law by more general equations. Further each heat conduction generalization turn out in constitution of generalized theory of thermoelasticity. As an example, Lord and Shulman [2] modified Fourier law of heat conduction. This model gives hyperbolic type heat conduction equation with finite speed of thermal wave propagation. Lord Shulman theory is also known as extended thermoelasticity. Also Green and Lindsay [6] referred to as a temperature rate dependent thermoelasticity, whereas Green and Naghdi [13] investigated the thermoelastic theory without energy dissipation. Sherief and Hamza [60] used Laplace transform technique to derive solution of one dimensional infinite long hollow cylinder under generalized thermoelasticity with one relaxation time.

But in this present article our study is based on the heat conduction equation with time-fractional differential operator and this operator successfully describes memory effect in real life situations. Hence due to physical application in real situation fractional calculus is used in various fields like engineering, physics, mathematics, geology, bioengineering, robotics etc. In [14, 26 and 45], a study includes modification of many existing physical processes based models by using fractional calculus. Caputo [1, 7] and Caputo and Mainardi [3, 4] studied the relationship between the theory of linear viscoelasticity and fractional derivative and found that the fractional model shows good agreement with experimental result. Thermoelastic problem of infinite cylinder with time-fractional diffusion-wave equation solved by applying Integral transform technique by
Povstenko [29]. The solution of non-axisymmetric time-fractional diffusion-wave equation in a cylindrical coordinates was determined by Povstenko [30]. A non-axisymmetric solution to time-fractional heat conduction equation in cylindrical coordinates for a half-space with source was evaluated by Povstenko [31]. Ezzat and Karamany [32, 33 and 34] constructed a new mathematical model of magnetothermoelasticity and electro-thermoelasticity by considering a new heat conduction law with timefractional order and applied to a perfect conducting half-space of elastic material respectively. Further, Ezzat [36] expand new mathematical model by considering fractional order heat conduction law of two-temperature for magneto-thermoelasticity and electromagnetic thermo fluid respectively.

Raslan [55] investigated the theory of thermoelasticity based on fractional order for a two dimensional thick plate with traction free lower and upper surface due to axisymmetric distributions of temperature. Sherief and Latief [43] found the application of one dimensional thermoelastic problem using the fractional calculus methodology in a half space. Xiong and Guo [59] investigated fractional order thermoelasticity for a one-dimensional finite length generalized magnetothermoelastic problem of a thermoelastic rod. In [35, 40, 41, 44, 56, 57 and 58], various thermoelastic problems studied which based on the theory of fractional-order thermoelastic model. In [61and 62], Transient hygrothermal response in a time-fractional problem was examined in a cylinder and sphere respectively. Sur and Kanoria [39, 49] studied two dimensional fractional order theories of thermoelastic with wave speed distribution and Functionally Graded Variable Material properties. A mathematical modeling of a circular disk due to partially distributed heat supply in context of fractional order theory of thermoelasticity was done by Kumar and Khobragade [63-64]. Thermoelastic deformation of a solid circular cylinder by application of fractional order theory was analyzed by Kumar and Khobragade [65-66]. Some other related significant contribution to fractional order theory has been discussed in [21- 23, 27, 38, 42, 50, 51 and 53].

From the above available literature it is noticed that maximum of the studies concern with steady state temperature distribution on nonhomogeneous cylinders with homogeneous material properties also heat production lead to various technical problems during mechanical applications. But from practical point of view thermoelastic problems with non homogeneous material properties are more realistic and having significant applications in high temperature conditions. Further analysis based on thermal stresses thermoelastic problems has significant growth in design of steam and gas turbines 
and nuclear reactors. Also the fractionalorder theory of thermoelasticity estimates a lagging response to physical stimulus. Hence proposed study for nonhomogeneous materials with internal heat generation in context of fractional order theory of thermoelasticity is useful for researchers working in material sciences, designers of new material structure where high thermal loading is considered and those working to further develop the basic theory of thermoelastic problems for non homogeneous structural materials by using fractional calculus approach. This problem concerned with the thermal behavior of a thick hollow cylinder with internal heat generation in context of fractional order theory of thermoelasticity.

In the present paper, we have assumed a $2 \mathrm{D}$ thermoelastic problem of a thick cylinder in context of fractional thermoelasticity in which sectional heating is applied on the curved surface. The material properties of cylinder except Poisson's ratio and density are considered to be nonhomogeneous given by a simple power law in axial direction. Numerical computations are obtained and examined for both the homogeneous and nonhomogeneous cases for hollow cylinders.

\section{Formulation of the problem}

We assume a nonhomogeneous thick hollow cylinder with internal heat generation of radius varying from $r=a$ to $r=b$ and thickness from $z=h_{1}$ to $z=h_{2}, \quad$ occupying the space $D=\left\{\begin{array}{l}(x, y, z) \in R^{3}: h_{1} \leq z \leq h_{2} \text { and } \\ a \leq\left(x^{2}+y^{2}\right)^{1 / 2} \leq b\end{array}\right\} \quad$ where $r=\left(x^{2}+y^{2}\right)^{1 / 2}$. The above thermoelastic problem is framed mathematically for a nonlocal Caputo type time fractional heat conduction equation of order $\alpha$ for nonhomogeneous thick hollow cylinder. The expression for Caputo type fractional derivative of function $f(t)$ is given as [21]

$$
\begin{aligned}
& \frac{d^{\alpha} f(t)}{d t^{\alpha}}=\frac{1}{\Gamma(n-\alpha)} \int_{0}^{t}(t-\tau)^{n-\alpha-1} \frac{d^{n} f(\tau)}{d \tau^{n}} d \tau, \\
& t>0, n-1<\alpha<n
\end{aligned}
$$

with the following Laplace transform rule, where Caputo derivative needs the initial values of the function $f(t)$ and its corresponding integral derivatives of order $k=1,2,3, \ldots, n-1$

$$
\begin{aligned}
& L\left\{\frac{d^{\alpha} f(t)}{d t^{\alpha}}\right\}=s^{\alpha} L\{\bar{f}(s)\}-\sum_{k=0}^{n-1} f^{(k)}\left(0^{+}\right) s^{\alpha-1-k}, \\
& n-1<\alpha<n .
\end{aligned}
$$

in which $s$ is the transform parameter and $n$ is a positive integer.

\section{Temperature distribution}

The governing transient heat conduction equation in the context of fractional-order theory subjected to a time dependent heat flux with internal heat generation for a two dimensional thermoelastic problem of a nonhomogeneous thick hollow cylinder satisfies the differential equation,

$$
\begin{gathered}
\frac{1}{r} \frac{\partial}{\partial r}\left(k(z) r \frac{\partial T}{\partial r}\right)+\frac{\partial}{\partial z}\left(k(z) \frac{\partial T}{\partial z}\right)+g(r, z, t) \\
=c(z) \rho \frac{\partial^{\alpha} T}{\partial t^{\alpha}}
\end{gathered}
$$

Using the physically convective heat exchange boundary conditions at a curved surface by following [52] written as

$$
\begin{gathered}
\left.\left(e_{1} T-k_{1} D_{R L}^{1-\alpha} \frac{\partial T}{\partial r}\right)\right|_{r=a}=0, h_{1} \leq z \leq h_{2}, t>0 \\
\left.\left(e_{2} T+k_{2} D_{R L}^{1-\alpha} \frac{\partial T}{\partial r}\right)\right|_{r=b}=Q_{1} \delta\left(z-z_{0}\right) \delta(t), \\
h_{1} \leq z \leq h_{2}, t>0
\end{gathered}
$$

$$
\begin{array}{ll}
\left.(T)\right|_{z=h_{1}}=0, & a \leq r \leq b, t>0 \\
\left.(T)\right|_{z=h_{2}}=0, & a \leq r \leq b, t>0
\end{array}
$$

And zero initial conditions are,

$$
\begin{array}{lll}
T=0, & \text { at } t=0, & 0<\alpha \leq 2 \\
\frac{\partial T}{\partial t}=0, & \text { at } t=0, & 1<\alpha \leq 2
\end{array}
$$

where $T(r, z, t)$ denote the temperature function of the hollow cylinder at any time $t, g(r, z, t)$ denote the heat generation function, $k(z)$ represent the thermal conductivity, $\rho$ refer for the constant density and $c(z)$ denote the calorific capacity of the material for the inhomogeneous region. Also $\delta\left(r-r_{0}\right)$ and $\delta\left(z-z_{0}\right)$ denote the Dirac Delta function having 
$a \leq r_{0} \leq b$ and $h_{1} \leq z_{0} \leq h_{2} ; Q_{1}$ are the heat flux with constant strength. Also $D_{R L}^{-\alpha} T(r, z, t)$ for $\alpha>0$ is the Riemann-Liouville fractional integral $I^{\alpha} T(r, z, t), e_{1}, e_{2}$ denote the external heat transfer coefficients $\& k_{1}, k_{2}$ denote the thermal conductivity coefficients.

\section{Displacements and thermal stresses}

For axisymmetric problem of two-dimensional thick cylinder the relation between strain displacements can be expressed as Hata [9]

$$
\begin{aligned}
& e_{r r}=\frac{\partial u}{\partial r}, e_{\theta \theta}=\frac{u}{r}, e_{z z}=\frac{\partial w}{\partial z}, \\
& e_{r z}=\frac{1}{2}\left(\frac{\partial u}{\partial z}+\frac{\partial w}{\partial r}\right)
\end{aligned}
$$

Where $u$ and $w$ are the displacement components in the radial and axial directions, respectively.

Stress-strain relationships are [9]

$$
\begin{aligned}
\sigma_{r r}= & 2 \mu(z) e_{r r}+\lambda(z) e \\
& -(3 \lambda(z)+2 \mu(z)) \alpha_{T}(z) T \\
\sigma_{\theta \theta}= & 2 \mu(z) e_{\theta \theta}+\lambda(z) e \\
& -(3 \lambda(z)+2 \mu(z)) \alpha_{T}(z) T \\
\sigma_{z z}= & 2 \mu(z) e_{z z}+\lambda(z) e \\
& -(3 \lambda(z)+2 \mu(z)) \alpha_{T}(z) T \\
\sigma_{r z}= & 2 \mu(z) e_{r z}
\end{aligned}
$$

Condition for equilibrium are given as

$$
\begin{aligned}
& \frac{\partial \sigma_{r r}}{\partial r}+\frac{\partial \sigma_{r z}}{\partial z}+\frac{\sigma_{r r}-\sigma_{\theta \theta}}{r}=0 \\
& \frac{\partial \sigma_{r z}}{\partial r}+\frac{\partial \sigma_{z z}}{\partial z}+\frac{\sigma_{r z}}{r}=0
\end{aligned}
$$

Where $e_{r r}, e_{\theta \theta}, e_{z z}$ in equation (11) to (14) denotes the strain Components, here $e=\left(e_{r r}+e_{\theta \theta}+e_{z z}\right)$ and $\quad \lambda(z)$ and $\mu(z)$ represents the Lame constants, also $\alpha_{T}(z)$ refers the coefficient of thermal expansion.

Further shear modulus $\mu(z)$ and coefficient of thermal expansion $\alpha_{T}(z)$ are assumed vary in the axial direction given as by following Hata [9],

$$
\mu(z)=\mu_{0} z^{p}, \quad \alpha_{T}(z)=\alpha_{0} z^{p}
$$

In above equation $\mu_{0}$ and $\alpha_{0}$ are reference values of shear modulus and coefficient of thermal expansion, also relation between Poisson's ratio $v$ and $p$ is related by the relation $p v=1-2 v$, where $v$ is constant and $p \geq 0$.

Equilibrium equation for displacement are obtained by using equations (10)-(14) in (15) and (16), as

$$
\begin{aligned}
& \Delta^{2} u-\frac{u}{r^{2}}+\frac{v}{(1-2 v)} \frac{\partial e}{\partial r}+\frac{e_{r z}}{\mu(z)} \frac{\partial \mu(z)}{\partial z} \\
& +2 \frac{\partial e_{r z}}{\partial z}-\frac{(1+v)}{(1-2 v)} \alpha_{T}(z) \frac{\partial T}{\partial r}=0, \\
& \Delta^{2} w+\frac{v}{(1-2 v)} \frac{\partial e}{\partial z}+\frac{\partial e_{r r}}{\partial z}+\frac{e_{z z}}{\mu(z)} \frac{\partial \mu(z)}{\partial z} \\
& +\frac{e}{2 \mu(z)} \frac{\partial \lambda(z)}{\partial z}-\frac{(1+v)}{(1-2 v)} \\
& \times\left[\alpha_{T}(z) \frac{\partial T}{\partial z}+T \frac{\partial \alpha_{T}(z)}{\partial z}\right] \\
& -\frac{\alpha_{T}(z)}{2 \mu(z)} T\left[3 \frac{\partial \lambda(z)}{\partial z}+2 \frac{\partial \mu(z)}{\partial z}\right]+\frac{1}{r} \frac{\partial u}{\partial z}=0
\end{aligned}
$$

where $\nabla^{2}$ is given by

$$
\nabla^{2}=\frac{\partial^{2}}{\partial r^{2}}+\frac{1}{r} \frac{\partial}{\partial r}+\frac{\partial^{2}}{\partial z^{2}}
$$

Without the action of body forces the solution of equation (17), (18) in the cylindrical coordinate system can be expressed by the Goodier's thermoelastic displacement potential $\phi$ and the Boussinesq harmonic functions $\varphi$ and $\psi$ following [17] as

$$
\begin{aligned}
& u=\frac{\partial \varphi}{\partial r}+\frac{\partial \phi}{\partial r}+z \frac{\partial \psi}{\partial r} \\
& w=\frac{\partial \varphi}{\partial z}+\frac{\partial \phi}{\partial z}+z \frac{\partial \psi}{\partial z}-(3-4 v) \psi
\end{aligned}
$$

Where $\phi, \varphi$ and $\psi$ must satisfy the following conditions

$$
\nabla^{2} \phi=K(z) \tau, \quad \nabla^{2} \varphi=0, \text { and } \nabla^{2} \psi=0
$$

Where restraint coefficient is 
$K(z)=\frac{(1+v)}{(1-v)} \alpha_{T}(z)$ and $\tau=T-T_{i}$, in which

$T_{i}$ denotes the surrounding temperature.

For sake of convenience take

$$
-\int(\varphi+z \psi) d z=M
$$

Now using (23) in equation (20) and (21), it gives Michell's function $M$ expressed instead of Boussinesq harmonic functions $\varphi$ and $\psi$ as below

$$
\begin{aligned}
& u=\frac{\partial \phi}{\partial r}-\frac{\partial^{2} M}{\partial r \partial z} \\
& w=\frac{\partial \phi}{\partial z}+2(1-v) \nabla^{2} M-\frac{\partial^{2} M}{\partial z^{2}}
\end{aligned}
$$

Where Michell's function must satisfy the condition given below

$$
\nabla^{2} \nabla^{2} M=0
$$

Now by using equation (24) and (25) in equations (11) to (14) and (17) to (18), the following results are obtained for thermoelastic fields as

$$
\begin{aligned}
& \Delta^{2} \phi-\frac{\partial}{\partial z}\left(\nabla^{2} M\right)-\frac{1}{r^{2}}\left(\phi-\frac{\partial M}{\partial z}\right) \\
& -\frac{(1+v)}{(1-2 v)} \alpha_{T}(z) T=0, \\
& \Delta^{2} \phi+2(1-v)\left[\nabla^{2} M+\frac{\partial}{\partial z}\left(\nabla^{2} M\right)+\frac{\partial}{\partial r}\left(\nabla^{2} M\right)\right] \\
& +2(1-v)\left[\frac{2-r}{r^{3}} \frac{\partial M}{\partial r}-\frac{2}{r^{2}} \frac{\partial^{2} M}{\partial r^{2}}\right] \\
& -\frac{(1+v)}{(1-2 v)} \alpha_{T}(z) T-(3 \lambda+2 \mu) \frac{\alpha_{T}(z)}{2 \mu(z)} T \\
& +\frac{1}{r}\left[\frac{\partial \phi}{\partial r}-\frac{\partial^{2} M}{\partial r \partial z}\right]=0,
\end{aligned}
$$

The corresponding stresses $\sigma_{r r}, \sigma_{\theta \theta}, \sigma_{z z}$ and $\sigma_{r z}$ are given by

$$
\begin{aligned}
& \sigma_{r r}=2 \mu(z) \frac{\partial}{\partial r}\left[\frac{\partial \phi}{\partial r}-\frac{\partial^{2} M}{\partial r \partial z}\right]+ \\
& \lambda(z)\left[\nabla^{2} \phi+(1-2 v) \frac{\partial}{\partial z}\left(\nabla^{2} M\right)\right] \\
& -(3 \lambda(z)+2 \mu(z)) \alpha_{T}(z) T
\end{aligned}
$$

$$
\begin{aligned}
& \sigma_{\theta \theta}=2 \mu(z) \frac{1}{r}\left[\frac{\partial \phi}{\partial r}-\frac{\partial^{2} M}{\partial r \partial z}\right] \\
& +\lambda(z)\left[\nabla^{2} \phi+(1-2 v) \frac{\partial}{\partial z}\left(\nabla^{2} M\right)\right] \\
& -(3 \lambda(z)+2 \mu(z)) \alpha_{T}(z) T \\
& \sigma_{z z}=2 \mu(z) \frac{\partial}{\partial z}\left(\frac{\partial \phi}{\partial z}+2(1-v) \nabla^{2} M-\frac{\partial^{2} M}{\partial z^{2}}\right) \\
& +\lambda(z)\left[\nabla^{2} \phi+(1-2 v) \frac{\partial}{\partial z}\left(\nabla^{2} M\right)\right] \\
& -(3 \lambda(z)+2 \mu(z)) \alpha_{T}(z) T \\
& \sigma_{r z}=\mu(z) \frac{\partial}{\partial z}\left[\frac{\partial \phi}{\partial r}-\frac{\partial^{2} M}{\partial r \partial z}\right] \\
& +\mu(z) \frac{\partial}{\partial r}\left(\frac{\partial \phi}{\partial z}+(1-2 v) \nabla^{2} M-\frac{\partial^{2} M}{\partial z^{2}}\right)
\end{aligned}
$$

For the traction free surface boundary conditions for stress functions are as

$$
\left.\begin{array}{lll}
\sigma_{r r}=0 & \text { at } & r=a \\
\sigma_{r r}=0 & \text { at } & r=b
\end{array}\right\}
$$

Above equations (3) to (33) represents the mathematical modeling of the time fractional thermoelastic problem of nonhomogeneous hollow cylinder with internal heat generation.

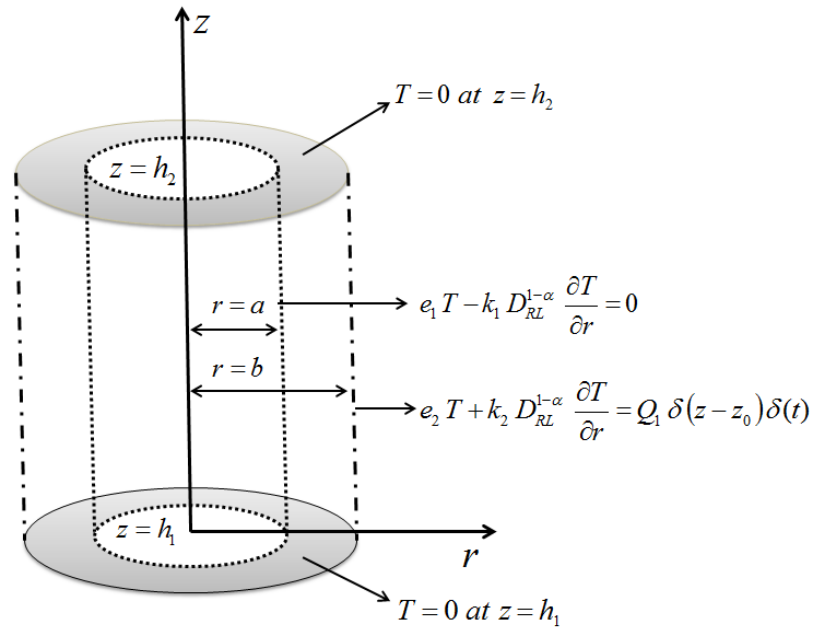

Figure 1. Geometry of nonhomogeneous hollow cylinder 


\section{Solution of the problem}

Solution of the heat conduction problem

From equation (3), and considering for sake of brevity as

$$
\begin{aligned}
& k(z)=k_{0} z^{p}, c(z)=c_{0} z^{p}, \rho=\rho_{0}, \\
& g(r, z, t)=k_{0} z^{((1+p) / 2)} g_{1}(r, t), \\
& g_{1}(r, t)=\delta\left(r-r_{0}\right) \delta\left(t-t_{0}\right)
\end{aligned}
$$

We get

$$
\begin{aligned}
& \left(\frac{\partial^{2} T}{\partial r^{2}}+\frac{1}{r} \frac{\partial T}{\partial r}\right)+\left(\frac{\partial^{2} T}{\partial z^{2}}+\frac{p}{z} \frac{\partial T}{\partial z}\right) \\
& +z^{((1-p) / 2)} g_{1}(r, t)=\frac{1}{\kappa} \frac{\partial^{\alpha} T}{\partial t^{\alpha}}
\end{aligned}
$$

\section{Where}

$$
\kappa=\left(k_{0} / c_{0} \rho_{0}\right)
$$

With the boundary and initial conditions as,

$$
\begin{aligned}
& \left.\left(e_{1} T-k_{1} D_{R L}^{1-\alpha} \frac{\partial T}{\partial r}\right)\right|_{r=a}=0, \\
& h_{1} \leq z \leq h_{2}, t>0 \\
& \left.\left(e_{2} T+k_{2} D_{R L}^{1-\alpha} \frac{\partial T}{\partial r}\right)\right|_{r=b}=Q_{1} \delta\left(z-z_{0}\right) \delta(t), \\
& h_{1} \leq z \leq h_{2}, t>0 \\
& \left.(T)\right|_{z=h_{1}}=0, a \leq r \leq b, t>0 \\
& \left.(T)\right|_{z=h_{2}}=0, a \leq r \leq b, t>0 \\
& T=0, \quad \text { at } t=0, \quad 0<\alpha \leq 2 \\
& \frac{\partial T}{\partial t}=0, \text { at } t=0, \quad 1<\alpha \leq 2
\end{aligned}
$$

Here the reference value of thermal conductivity is denoted as $k_{0}$, calorific capacity as $c_{0}$ and density by $\rho_{0}$ respectively.

In order to remove $p$ from the numerator of equation (35), we use the following variable transformation as $T=z^{((1-p) / 2)} \theta(r, z, t)$

Hence, equation (35) becomes

$$
\begin{aligned}
& \left(\frac{\partial^{2} \theta}{\partial r^{2}}+\frac{1}{r} \frac{\partial \theta}{\partial r}\right)+\left(\frac{\partial^{2} \theta}{\partial z^{2}}+\frac{1}{z} \frac{\partial \theta}{\partial z}+\frac{\gamma^{2}}{z^{2}} \theta\right) \\
& +g_{1}(r, t)=\frac{1}{\kappa} \frac{\partial^{\alpha} \theta}{\partial t^{\alpha}}
\end{aligned}
$$

here $\gamma^{2}=(1-p) / 2$

The initial and boundary conditions are

$$
\begin{aligned}
& \left.\left(e_{1} \theta-k_{1} D_{R L}^{1-\alpha} \frac{\partial \theta}{\partial r}\right)\right|_{r=a}=0 \\
& h_{1} \leq z \leq h_{2}, t>0 \\
& \left.\left(e_{2} \theta+k_{2} D_{R L}^{1-\alpha} \frac{\partial \theta}{\partial r}\right)\right|_{r=b}=Q_{1} z^{((p-1) / 2)} \delta\left(z-z_{0}\right) \delta(t) \\
& , h_{1} \leq z \leq h_{2}, t>0 \\
& \left.(\theta)\right|_{z=h_{1}}=0, a \leq r \leq b, t>0 \\
& \left.(\theta)\right|_{z=h_{2}}=0, a \leq r \leq b, t>0 \\
& \theta=0, \quad \text { at } t=0, \quad 0<\alpha \leq 2 \\
& \frac{\partial \theta}{\partial t}=0, \text { at } t=0, \quad 1<\alpha \leq 2
\end{aligned}
$$

In order to solve the differential equation (42) we use the extended integral transform defined in Al-Hajri and Kalla [18] of order $i$ over the variable $Z$ as

$$
T\left[f(z), a, b ; \gamma_{i}\right]=\bar{f}\left(\gamma_{i}\right)=\int_{h_{1}}^{h_{2}} z f(z) S\left(\gamma_{i} z\right) d z
$$

where $S\left(\gamma_{i} z\right)$ represent the kernel of the integral transform (49) given as

$S\left(\gamma_{i} z\right)=Z_{i} \cos \left(\gamma_{i} \log z\right)-W_{i} \sin \left(\gamma_{i} \log z\right)$, $z>0$ and $\gamma_{i}(i=1,2,3, \ldots$.

$Z_{i}=\sin \left(\gamma_{i} \log h_{1}\right)+\sin \left(\gamma_{i} \log h_{2}\right)$

$W_{i}=\cos \left(\gamma_{i} \log h_{1}\right)+\cos \left(\gamma_{i} \log h_{2}\right)$,

and $\gamma_{i}$ denotes the real and positive roots of the transcendental equation

$\sin \left(\gamma \log h_{1}\right) \cos \left(\gamma \log h_{2}\right)$

$-\sin \left(\gamma \log h_{2}\right) \cos \left(\gamma \log h_{1}\right)=0$ 
The inversion transformation is given as

$$
f(z)=\sum_{i=1}^{\infty} \frac{\bar{f}\left(\gamma_{i}\right)}{S\left(\gamma_{i}\right)} S\left(\gamma_{i} z\right)
$$

here

$$
\int_{h_{1}}^{h_{2}} z S\left(\gamma_{i} z\right) S\left(\gamma_{j} z\right) d z= \begin{cases}S\left(\gamma_{i}\right), & i=j, \\ 0, & i \neq j .\end{cases}
$$

Hence, equations (42) to (49) become

$$
\begin{aligned}
& \left(\frac{\partial^{2} \bar{\theta}}{\partial r^{2}}+\frac{1}{r} \frac{\partial \bar{\theta}}{\partial r}\right)-\gamma_{i}^{2} \bar{\theta}+\bar{g}_{1}(r, t)=\frac{1}{\kappa} \frac{\partial^{\alpha} \bar{\theta}}{\partial t^{\alpha}} \\
& \left.\left(e_{1} \bar{\theta}-k_{1} D_{R L}^{1-\alpha} \frac{\partial \bar{\theta}}{\partial r}\right)\right|_{r=a}=0 \\
& h_{1} \leq z \leq h_{2}, t>0 \\
& \left.\left(e_{2} \bar{\theta}+k_{2} D_{R L}^{1-\alpha} \frac{\partial \bar{\theta}}{\partial r}\right)\right|_{r=b}=Q_{1} g_{0} \delta(t) \\
& h_{1} \leq z \leq h_{2}, t>0 \\
& \bar{\theta}=0, \quad \text { at } t=0, \quad 0<\alpha \leq 2 \\
& \frac{\partial \bar{\theta}}{\partial t}=0, \text { at } t=0, \quad 1<\alpha \leq 2 \\
& \text { W } t=0
\end{aligned}
$$

Where

$$
\begin{aligned}
& g_{0}=\int_{h_{1}}^{h_{2}} z^{((p+1) / 2)} \delta\left(z-z_{0}\right) S\left(\gamma_{j} z\right) d z \\
& \bar{g}_{1}(r, t)=\delta\left(r-r_{0}\right) \delta\left(t-t_{0}\right)
\end{aligned}
$$

By using the integral transform given in Al-Hajri and Kalla [18], the equations (54) and the boundary conditions given by equation (55) to (56), we get

$$
\begin{aligned}
& \frac{\partial^{\alpha} \overline{\bar{\theta}}}{\partial t^{\alpha}}+A_{1} \overline{\bar{\theta}}=A_{2}+A_{3} \delta\left(t-t_{0}\right), \\
& \overline{\bar{\theta}}=0, \quad \text { at } t=0, \quad 0<\alpha \leq 2 \\
& \frac{\partial \bar{\theta}}{\partial t}=0, \quad \text { at } t=0, \quad 1<\alpha \leq 2
\end{aligned}
$$

Where

$$
\begin{aligned}
& A_{1}=\kappa\left(q_{n}^{2}+\gamma_{i}^{2}\right), A_{2}=\frac{\kappa h_{1}}{k_{2}} M\left(q_{n} h_{1}\right) Q_{1} g_{0}, \\
& A_{3}=\kappa r_{0} M\left(q_{n} r_{0}\right)
\end{aligned}
$$

Here, $M\left(q_{n} r\right)$ denotes the kernel of the transform as

$$
\begin{aligned}
& M\left(q_{n} r\right)=\left[B\left(q_{n} a, e_{1}, k_{1}\right)+B\left(q_{n} b, e_{2}, k_{2}\right)\right] J_{0}\left(q_{n} r\right) \\
& -\left[A\left(q_{n} a, e_{1}, k_{1}\right)+A\left(q_{n} b, e_{2}, k_{2}\right)\right] Y_{0}\left(q_{n} r\right)
\end{aligned}
$$

Where

$$
\begin{aligned}
& A\left(q_{n} r, e_{n}, k_{n}\right)=e_{n} J_{0}\left(q_{n} r\right)+k_{n} q_{n} J_{0}^{\prime}\left(q_{n} r\right) ; \\
& n=1,2 ; r=a, b \\
& B\left(q_{n} r, e_{n}, k_{n}\right)=e_{n} Y_{0}\left(q_{n} r\right)+k_{n} q_{n} Y_{0}^{\prime}\left(q_{n} r\right) ; \\
& n=1,2 ; r=a, b
\end{aligned}
$$

Here $J_{0}$ is Bessel's function of first kind and $Y_{0}$ is of second kind, respectively and $q_{n}$ are the positive roots of the transcendental equation.

$$
\begin{aligned}
& B\left(q_{n} a, e_{1}, k_{1}\right) \times A\left(q_{n} b, e_{1}, k_{2}\right) \\
& -A\left(q_{n} a, e_{1}, k_{1}\right) \times B\left(q_{n} b, e_{2}, k_{2}\right)=0
\end{aligned}
$$

On using Laplace transform and its inversion to equation (59) by using the initial condition (60) and (61), we get

$$
\begin{aligned}
& \overline{\bar{\theta}}(n, t)=A_{2} t^{\alpha-1} E_{\alpha, \alpha}\left(-A_{1} t^{\alpha}\right) \\
& +A_{3}\left(t-t_{0}\right)^{\alpha-1} E_{\alpha, \alpha}\left(-A_{1}\left(t-t_{0}\right)^{\alpha}\right) \theta^{*}\left(t-t_{0}\right)
\end{aligned}
$$

Here $\theta^{*}\left(t-t_{0}\right)$ denotes the Heaviside Theta function and is given as

$$
\theta^{*}\left(t-t_{0}\right)= \begin{cases}0, & t<t_{0}, \\ 1, & t>t_{0},\end{cases}
$$

Applying inverse transform on equation (62), we obtain

$$
\begin{aligned}
& \bar{\theta}(r, t)=\left(\sum_{n=1}^{\infty} \frac{A_{2} t^{\alpha-1} E_{\alpha, \alpha}\left(-A_{1} t^{\alpha}\right)}{M\left(q_{n}\right)}\right. \\
& \sum_{n=1}^{\infty} \frac{A_{3}\left(t-t_{0}\right)^{\alpha-1} E_{\alpha, \alpha}\left(-A_{1}\left(t-t_{0}\right)^{\alpha}\right) \theta^{*}\left(t-t_{0}\right)}{M\left(q_{n}\right)} M\left(q_{n} r\right)
\end{aligned}
$$

Now, using the inverse transform defined in equation (52) to the equation (64), we obtain 
$\theta(r, z, t)=\sum_{i=1}^{\infty} \sum_{n=1}^{\infty} \frac{\bar{\theta}(r, t)}{S\left(\gamma_{i}\right)} \times S\left(\gamma_{i} z\right), z>0$

On using equation (65) in the relation $T=z^{(1-p) / 2} \theta(r, z, t)$, we obtain the required expression of temperature distribution function as

$$
\begin{aligned}
& T(r, z, t)=\sum_{i=1}^{\infty} \sum_{n=1}^{\infty}\left\{\xi_{1} \times \xi_{2}(t)\right. \\
& \left.\times\left[\xi_{3} J_{0}\left(q_{n} r\right)-\xi_{4} Y_{0}\left(q_{n} r\right)\right] \times g_{1}(z)\right\}
\end{aligned}
$$

where

$$
\begin{aligned}
& g_{1}(z)=z^{(1-p) / 2} \times S\left(\gamma_{i} z\right) \\
& \xi_{1}=1 / M\left(q_{n}\right) \times S\left(\gamma_{i}\right) \\
& \xi_{2}(t)=A_{2} t^{\alpha-1} E_{\alpha, \alpha}\left(-A_{1} t^{\alpha}\right) \\
& +A_{3}\left(t-t_{0}\right)^{\alpha-1} E_{\alpha, \alpha}\left(-A_{1}\left(t-t_{0}\right)^{\alpha}\right) \theta^{*}\left(t-t_{0}\right) \\
& \xi_{3}=\left[B\left(q_{n} h_{1}, e_{1}, k_{1}\right)+B\left(q_{n} h_{2}, e_{2}, k_{2}\right)\right], \\
& \xi_{4}=\left[A\left(q_{n} h_{1}, e_{1}, k_{1}\right)+A\left(q_{n} h_{2}, e_{2}, k_{2}\right)\right]
\end{aligned}
$$

\section{Thermoelastic equations}

The expression of Goodier's thermoelastic displacement potential governed by equation (22) is obtained by referring to the heat conduction equation (35) and its solution given by equation (66) as

$$
\begin{aligned}
& \phi=\sum_{i=1}^{\infty} \sum_{n=1}^{\infty} \frac{1}{\left[-q_{n}^{2} g_{1}(z)+g_{1}^{\prime \prime}(z)\right]} \\
& \times\left\{K(z)\left\{\xi_{1} \xi_{2}(t)\left[\xi_{3} J_{0}\left(q_{n} r\right)-\xi_{4} Y_{0}\left(q_{n} r\right)\right] g_{1}^{2}(z)\right\}\right. \\
& \left.-\frac{K(z) T_{i}(r, z)}{\xi_{1} \times \xi_{2}(t) \times\left[\xi_{3} J_{0}\left(q_{n} r\right)-\xi_{4} Y_{0}\left(q_{n} r\right)\right]}\right\}
\end{aligned}
$$

We assume the solution for Michell's function so as to satisfy the governed condition equation (26), as

$$
\begin{aligned}
& M=\sum_{i=1}^{\infty} \sum_{n=1}^{\infty}\left\{z^{((1-\beta) / 2)} \times[\cos (\log z)-\sin (\log z)]\right\} \\
& \times \omega^{n} t^{n-\alpha} E_{1, n-\alpha+1}(\omega t)\left[C_{m} J_{0}\left(q_{n} r\right)+D_{m} Y_{0}\left(q_{n} r\right)\right]
\end{aligned}
$$

Where $C_{m}$ and $D_{m}$ denotes constants.
Now, substitute the values of $\phi$ and $M$ in equation (24) and (25) the expression for displacement components obtained as

$$
\begin{aligned}
& u=\sum_{i=1}^{\infty} \sum_{n=1}^{\infty}\left\{\frac{\partial \phi}{\partial r}-q_{n} z^{-\frac{(1+\beta)}{2}}\left[\begin{array}{l}
\frac{(1+\beta)}{2} \cos (\log z) \\
+\frac{(3-\beta)}{2} \sin (\log z)
\end{array}\right]\right. \\
& \times \omega^{n} t^{n-\alpha} E_{1, n-\alpha+1}(\omega t) \times\left[C_{m} J_{1}\left(q_{n} r\right)+D_{m} Y_{1}\left(q_{n} r\right)\right]
\end{aligned}
$$

$$
\begin{aligned}
& w=\sum_{i=1}^{\infty} \sum_{n=1}^{\infty}\left\{\frac{\partial \phi}{\partial z}+(2 v-2) q_{n}^{2}\left[\begin{array}{l}
g_{2}(z) \exp (\omega t) \\
\times\left(C_{m} J_{0}\left(q_{n} r\right)+D_{m} Y_{0}\left(q_{n} r\right)\right)
\end{array}\right]\right. \\
& +\frac{(1-2 v)}{4}\left[z^{-\frac{(\beta+3)}{2}}\left[\begin{array}{c}
(\beta+5)(\beta-1) \cos (\log z) \\
-(\beta+1)(\beta-5) \sin (\log z)
\end{array}\right]\right\} \\
& \left.\times \omega^{n} t^{n-\alpha} E_{1, n-\alpha+1}(\omega t)\right] \\
& \times\left(C_{m} J_{0}\left(q_{n} r\right)+D_{m} Y_{0}\left(q_{n} r\right)\right)
\end{aligned}
$$

The expression of components of stresses can be obtained by using the displacement components given by equations (69) and (70) in equation (29) to (32). Also the values of constants $C_{m}$ and $D_{m}$ can be determined by using the traction free boundary conditions given by equation (33). We have not mentioned the large mathematical equations of stresses and constants, However numerical computations are carried out by using Mathematica software.

\section{Numerical calculations}

Mixtures of Copper and Tin metals assumed for numerical computations in the ratio 70:30 respectively, with non-dimensional variables are as [8] given below:

$$
\begin{aligned}
& T^{*}=\frac{T}{T_{R}}, \quad r^{*}=\frac{r}{a}, \quad z^{*}=\frac{z-h_{1}}{a}, t^{*}=\frac{\kappa t}{a^{2}}, \\
& h^{*}=\frac{h}{a}, \quad u^{*}=\frac{u}{\alpha_{0} T_{R} a}, \quad w^{*}=\frac{w}{\alpha_{0} T_{R} a}, \\
& \sigma_{r r}^{*}=\frac{\sigma_{r r}}{\alpha_{0} E \theta_{R}}, \quad \sigma_{\theta \theta}{ }^{*}=\frac{\sigma_{\theta \theta}}{\alpha_{0} E \theta_{R}}, \\
& \sigma_{z z}^{*}=\frac{\sigma_{z z}}{\alpha_{0} E \theta_{R}}, \quad \sigma_{r z}^{*}=\frac{\sigma_{r z}}{\alpha_{0} E \theta_{R}},
\end{aligned}
$$

The constants used during the numerical calculation are given as:

Inner radius of a cylinder $a=1 \mathrm{~cm}$, Outer radius of a cylinder $b=2 \mathrm{~cm}, t_{0}=2 \mathrm{sec}$, Thickness of cylinder 
$h_{1}=2 \mathrm{~cm}, \quad$ Thickness of cylinder $h_{2}=5 \mathrm{~cm}$, $r_{0}=1.5 \mathrm{~cm}$ and Reference temperature $T_{R}=32^{\circ} \mathrm{C}$

For the forgoing analysis mathematical simplicities are done by setting the radiation coefficients constants as $k_{1}=0.86, k_{2}=0.86$, and the convective heat transfer coefficients $e_{1}=1, e_{2}=1$

The other associated values are taken as:

Thermal diffusivity $\kappa=1.11 \mathrm{~cm}^{2} / \mathrm{s}^{\alpha}$, Coefficient of linear thermal expansion $\alpha_{0}=17 \times 10^{-6} /{ }^{0} C$, Young's modulus $E=4.41 \times 10^{7} \mathrm{~N} / \mathrm{cm}^{2}$, the relation between the parameter $p$, the Poisson's ratio $v$ and Shear modulus $\mu_{0}$ is $p=\frac{1-2 v}{v}$ and $\mu_{0}=\frac{E}{2(1+v)}$
Also for sake of simplicity we set: $Q_{1}=z^{2} \times\left(z^{2}-h_{1}^{2}\right)^{2} \times\left(z^{2}-h_{2}^{2}\right)^{2}$

Case 1: Homogeneous Case: $p=0, v=0.5$ and $\mu_{0}=1.47 \times 10^{7} \mathrm{~N} / \mathrm{cm}^{2}$

Case 2: Nonhomogeneous Case: $p=1.5$, $v=0.286$ and $\mu_{0}=1.715 \times 10^{7} \mathrm{~N} / \mathrm{cm}^{2}$

\section{Analysis of numerical Solutions}

The obtained mathematical results of temperature distribution, radial stress distribution, tangential stress distribution, axial stress and shear stress distribution for fractional-order parameter $\alpha=0.5, \alpha=1, \alpha=1.5, \alpha=2 \quad$ (depicting weak, normal and strong conductivity) computed numerically by MATEMATICA software for the finite hollow cylinder.
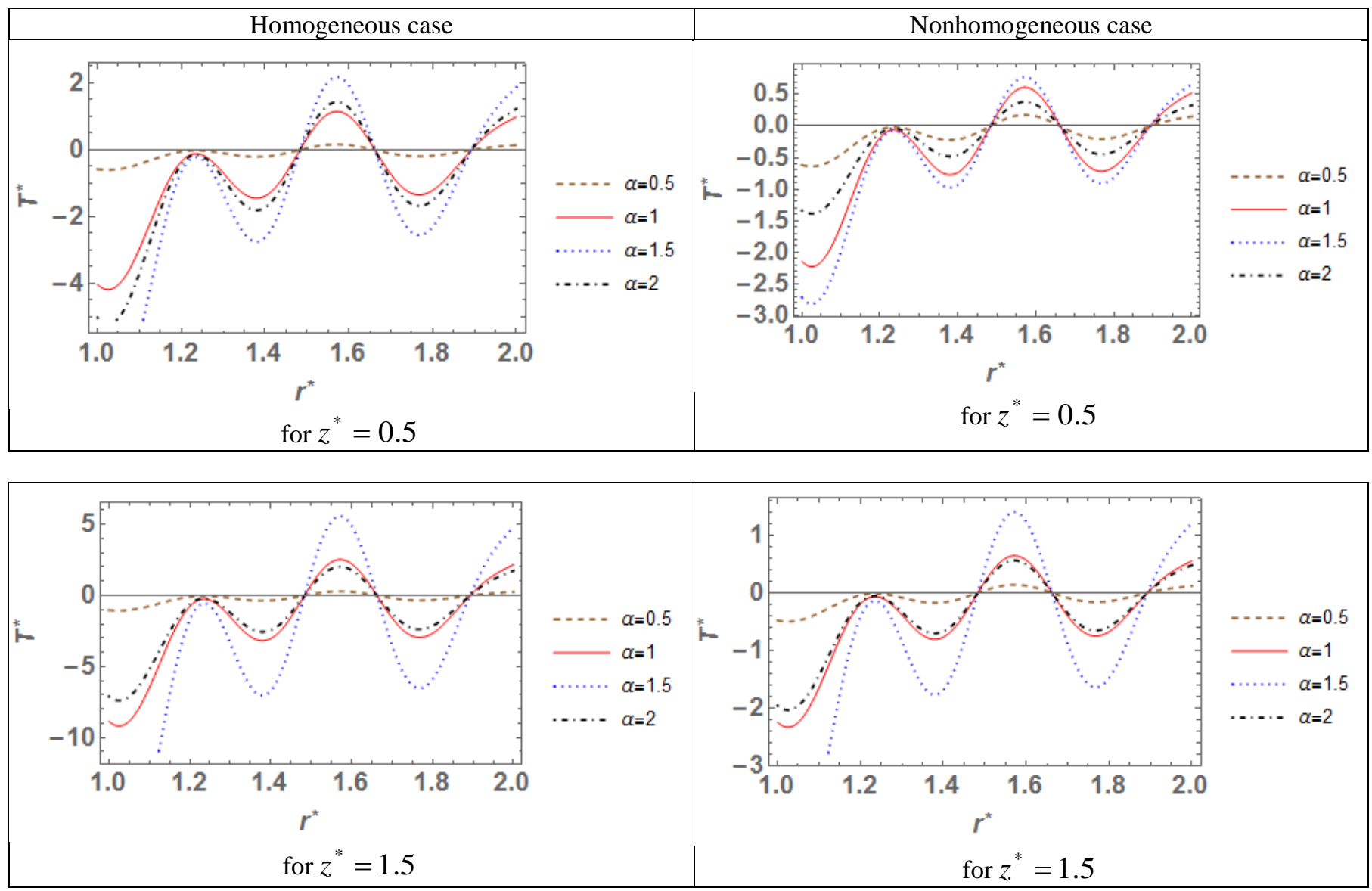


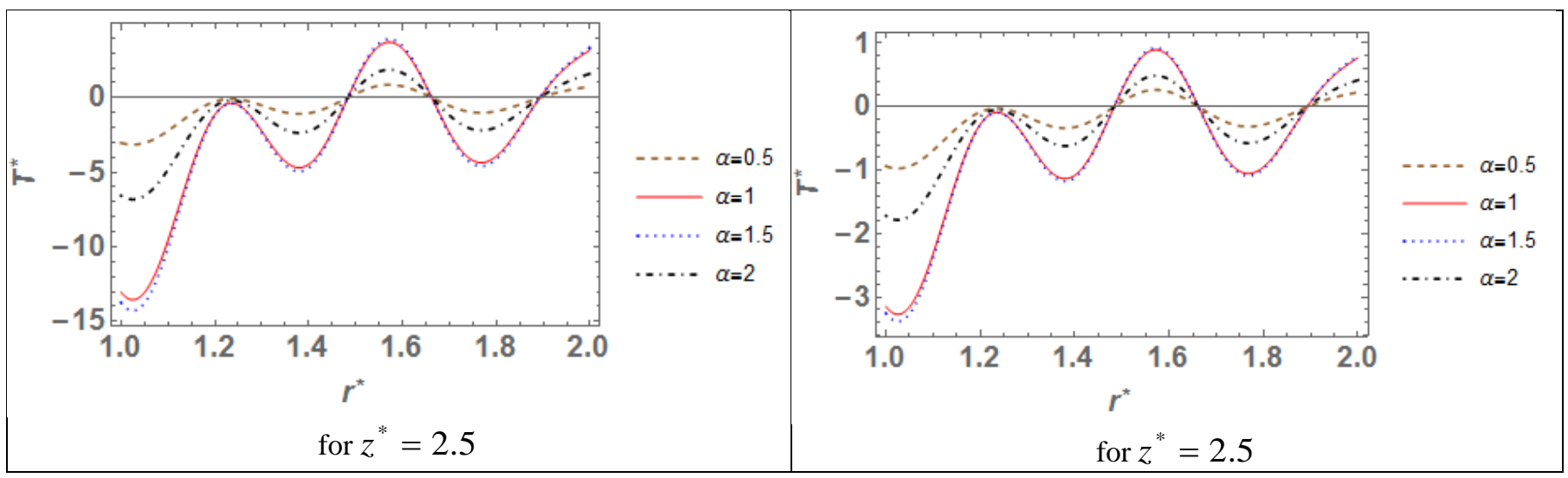

Figure 2. dimensionless temperature distribution function

Figure 2 represents the dimensionless temperature distribution $T^{*}$ along the radial direction of thick cylinder for different values of the fractional-order parameter $\alpha$ with different values of dimensionless thickness $z^{*}=0.5, \quad z^{*}=1.5$ and $\quad z^{*}=2.5$ for both homogeneous and non homogeneous cylinder. Above figure represents that initially temperature increases in region $1 \leq r \leq 1.2$ and after $r=1.2$ it becomes sinusoidal in nature. At both the radii ends $r^{*}=1$ and $r^{*}=2$ the temperature found nonzero value which is due to the action of internal heat generation and subjected sectional heating on the outer curved surface in both the homogeneous and nonhomogeneous cases. Also the values of the temperature follow a uniform pattern with respect to radius. For homogeneous case the magnitude of temperature distribution for cylinder is found high as compared to nonhomogeneous case. The magnitude of temperature is high at the lower surface because of sectional heating and is gradually goes on decreasing towards the upper surface. Further it is analyzed that the speed of thermal signals propagation varying directly proportional to the values of the different values of fractional-order parameter $\alpha$.

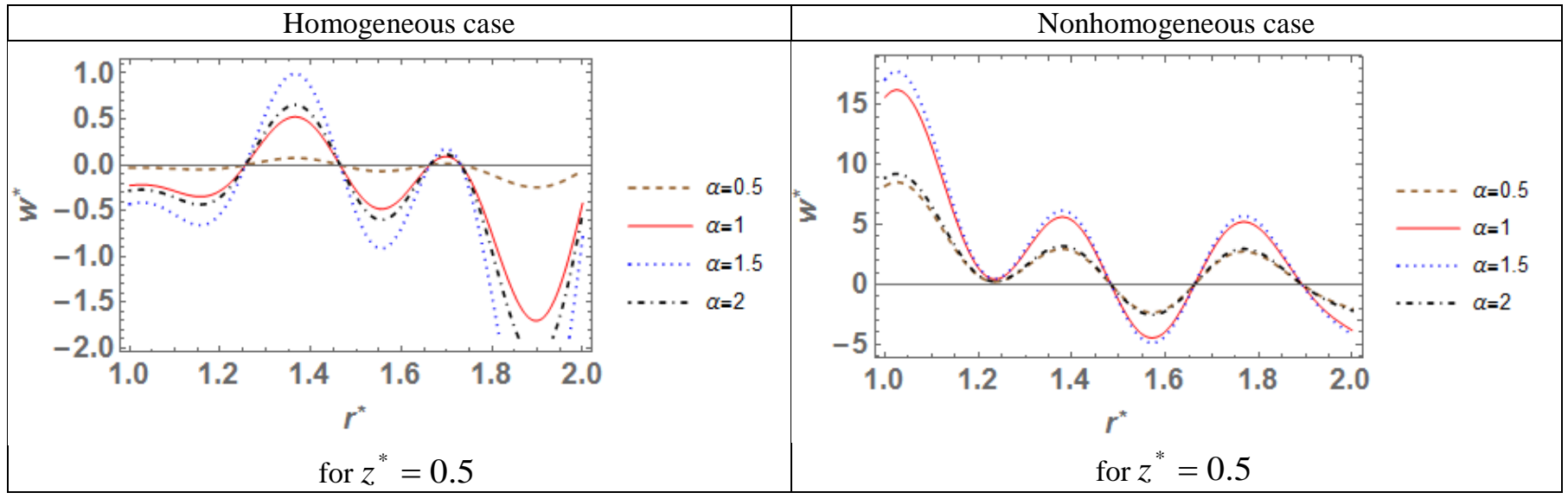



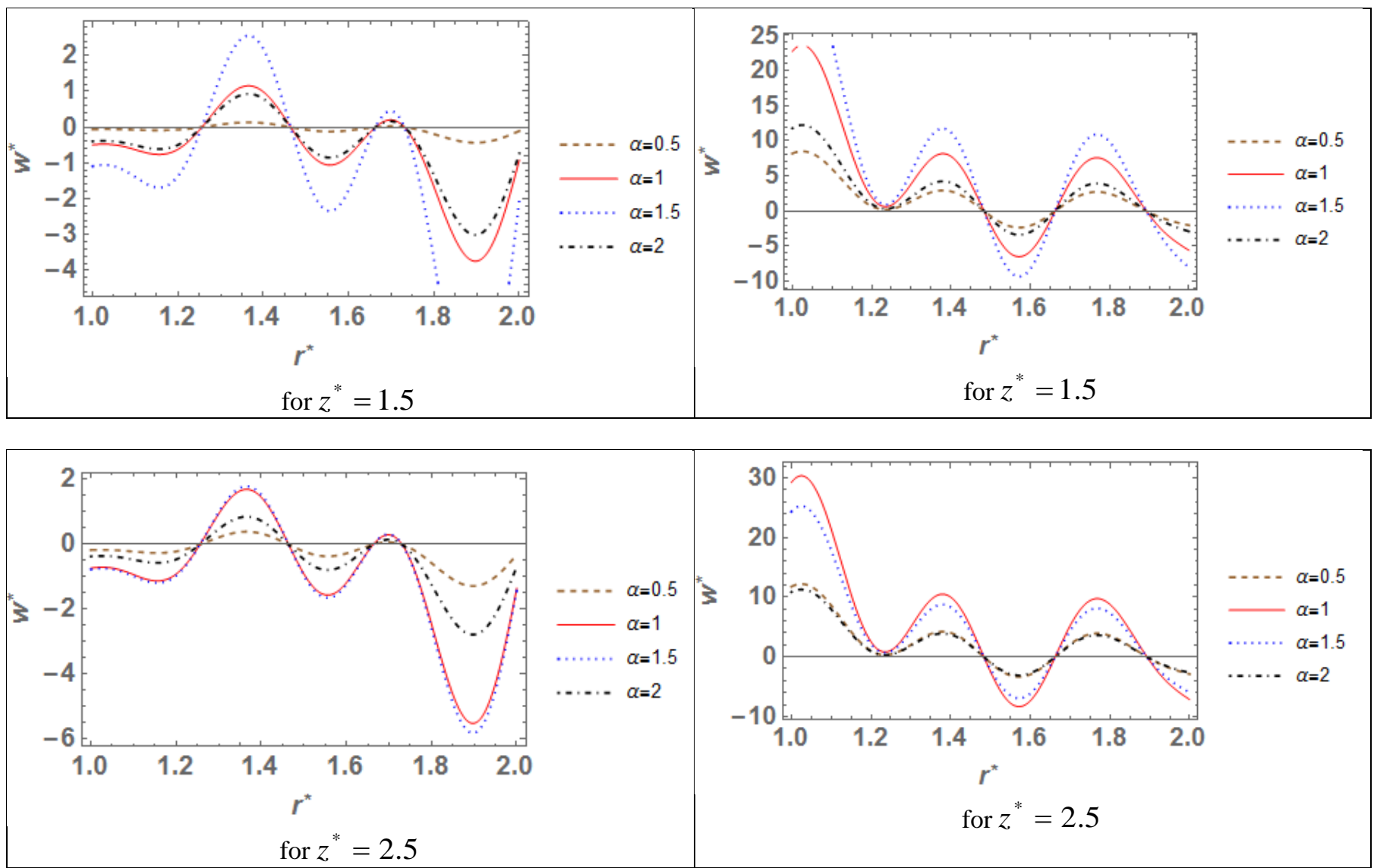

Figure 3. Shows dimensionless displacement distributions

Figure 3 represents dimensionless displacement $w^{*}$ variation along the radial direction $r^{*}$ for different values of fractional-order parameter $\alpha=0.5$, $\alpha=1, \alpha=1.5, \alpha=2$ with different values of dimensionless thickness $z^{*}=0.5, \quad z^{*}=1.5$ and $z^{*}=2.5$ for both homogeneous and non homogeneous cylinder. It is seen that the displacement distribution is more at the inner radius for nonhomogeneous as compare to homogeneous cylinder and is fluctuating in the region uniformly for both homogeneous and nonhomogeneous cases. Also propagation of the displacement functions is directly proportional to the values of the fractional-order parameter $\alpha$.

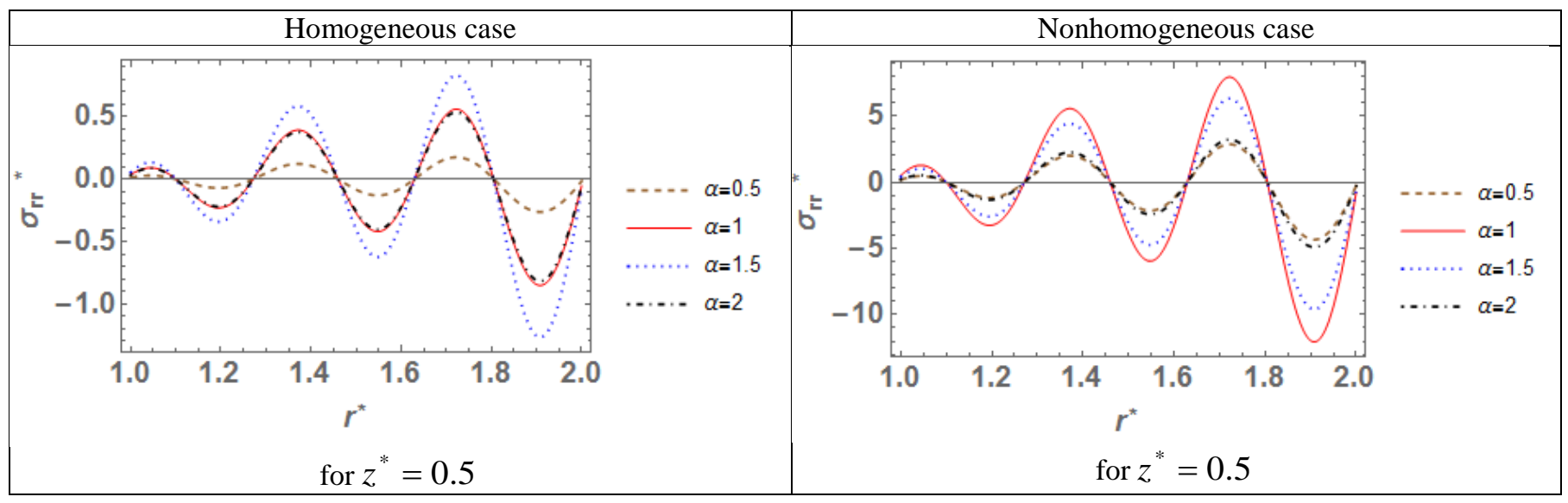



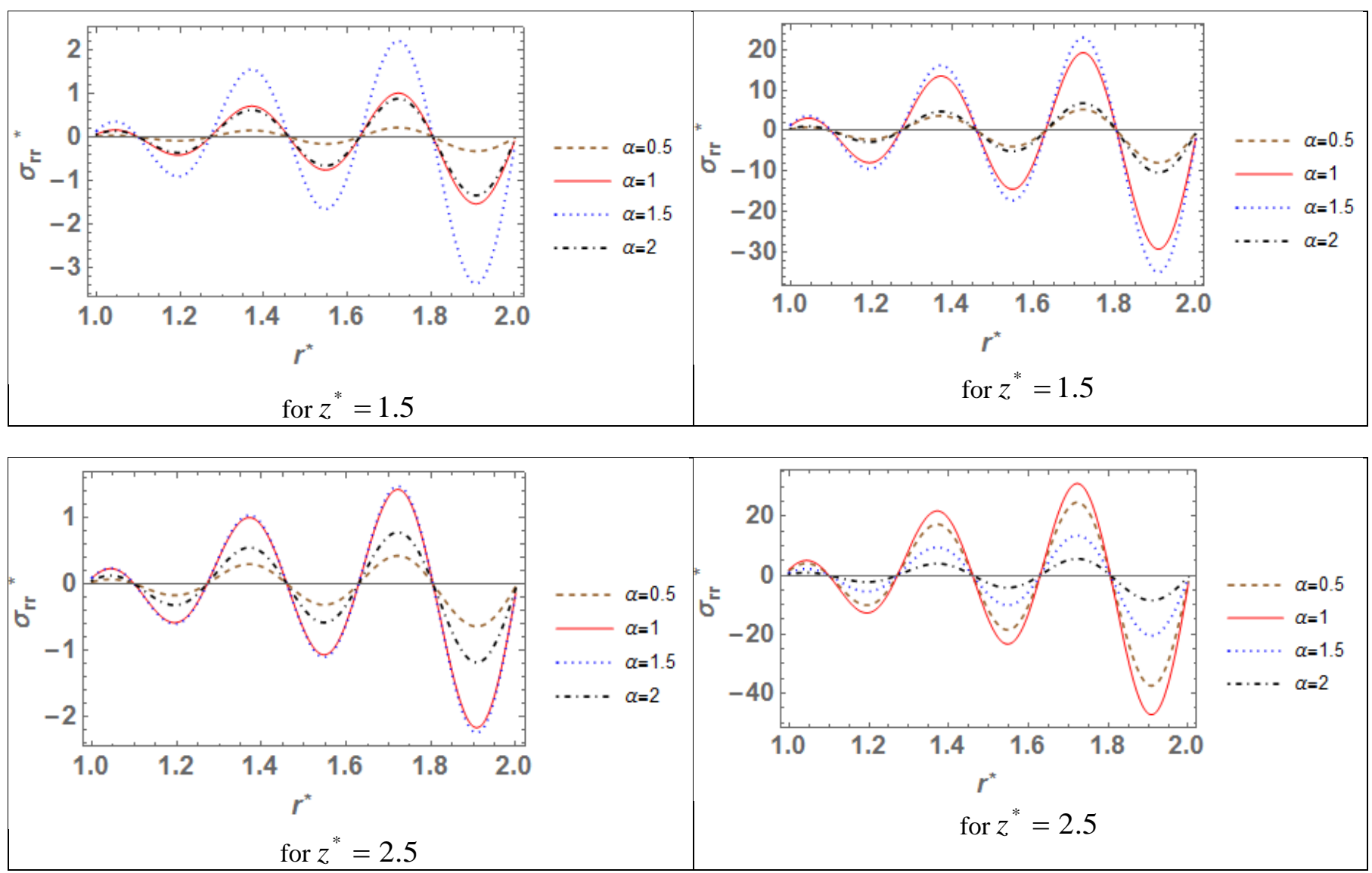

Figure 4. shows dimensionless radial stress distribution

Figure 4 represents the variation of dimensionless radial stress $\sigma_{r r}{ }^{*}$ in radial direction $r^{*}$ for different values of fractional order parameter with different values of dimensionless thickness $z^{*}=0.5$, $z^{*}=1.5$ and $z^{*}=2.5$ for both homogeneous and non homogeneous cylinder. It is seen that the value of radial stress is zero at both the radial ends

$r^{*}=1$ and $r^{*}=2$,

which is for both homogeneous and nonhomogeneous cylinders that's clearly agrees with the prescribed traction free boundary conditions. It is found that thickness directly effects stress variation that is for large value of $z^{*}$, small distribution of $\sigma_{r r}{ }^{*}$ attains, Also propagation of the radial stress functions is found directly proportional to the values of the fractionalorder parameter $\alpha$.

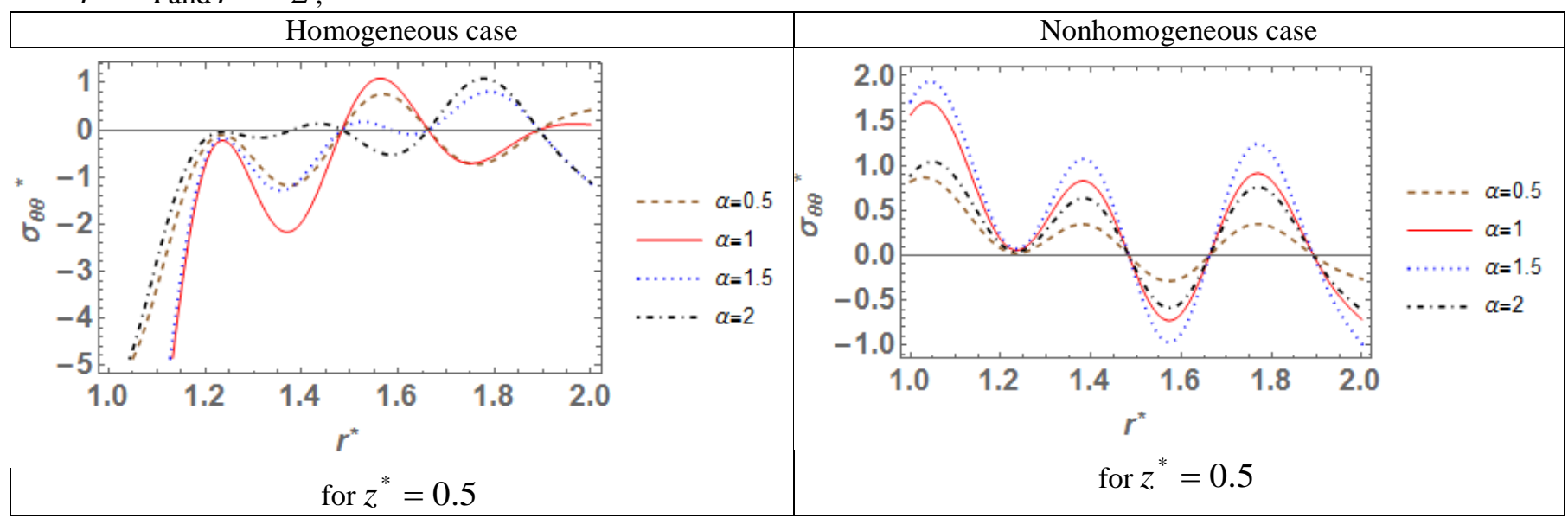



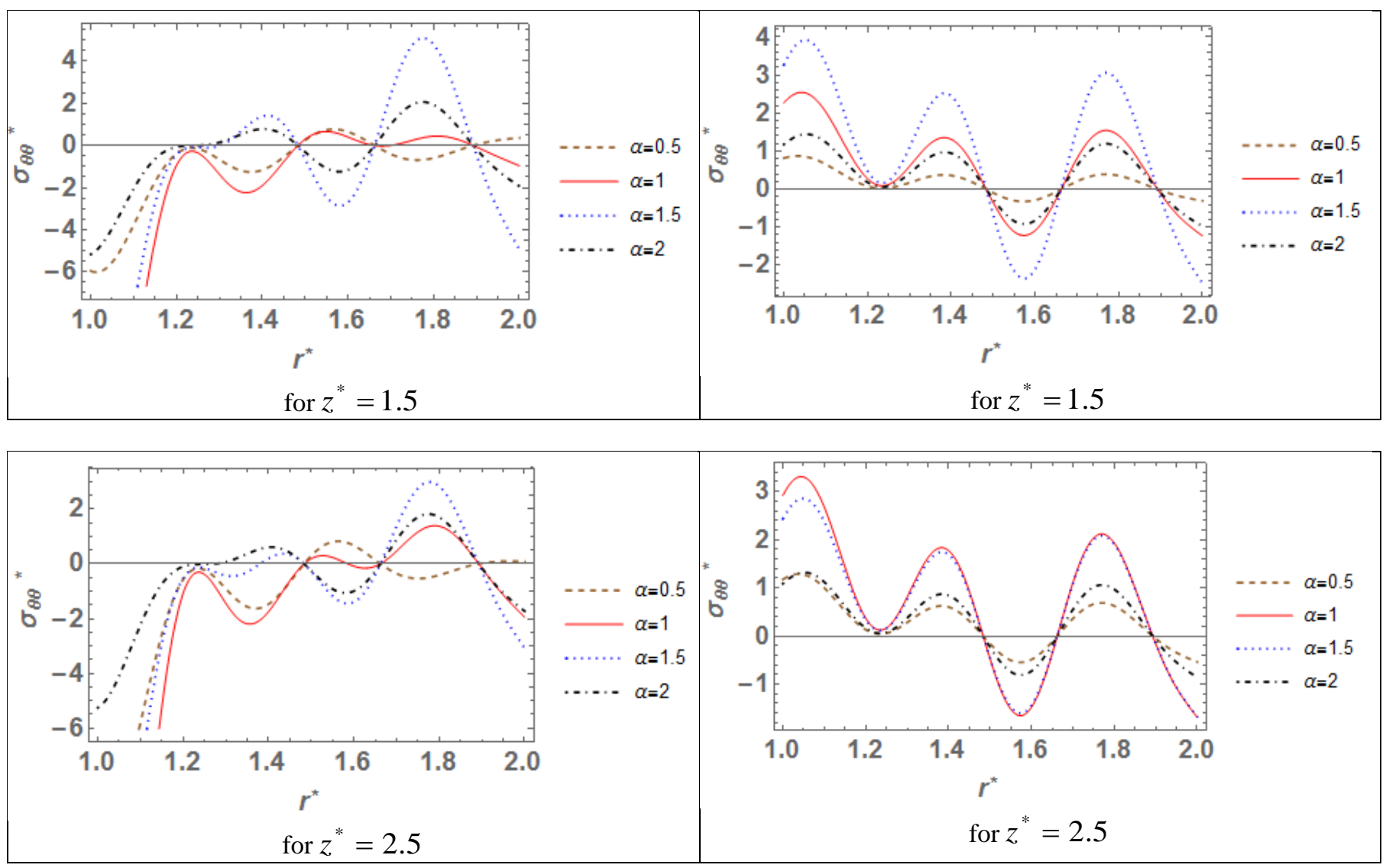

Figure 5. dimensionless tangential stress distributions

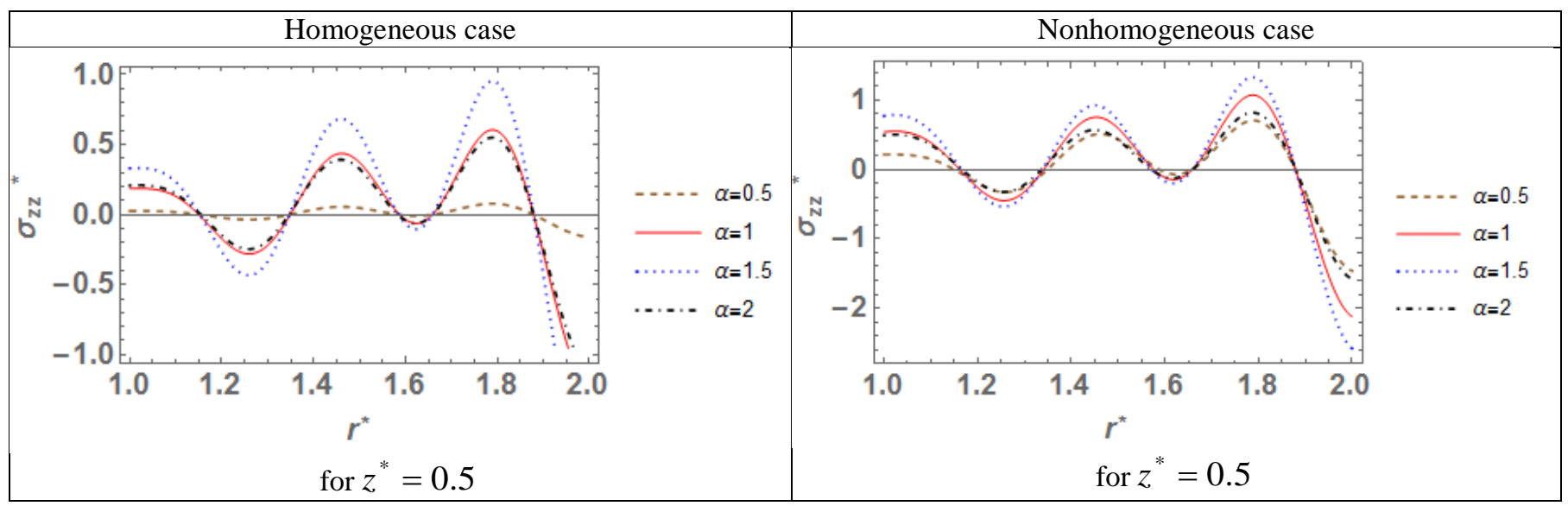



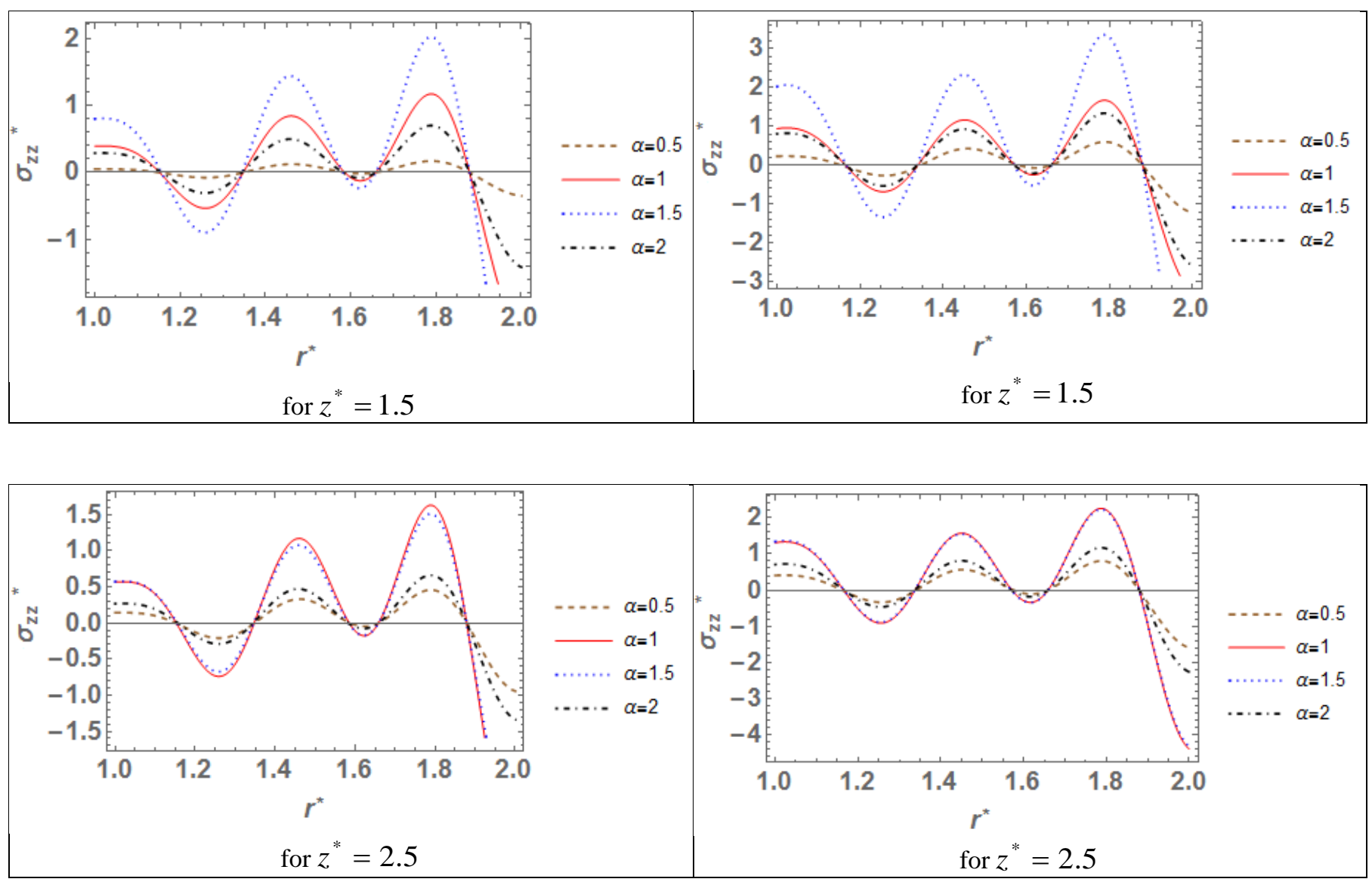

Figure 6. Dimensionless axial stress distributions

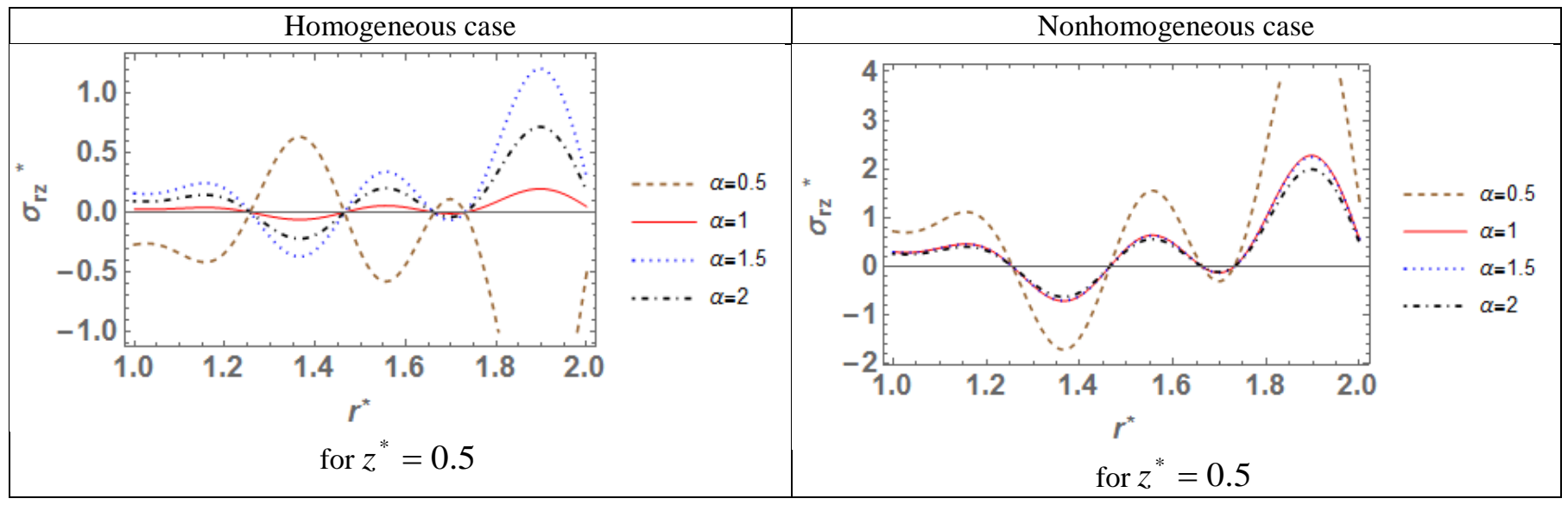



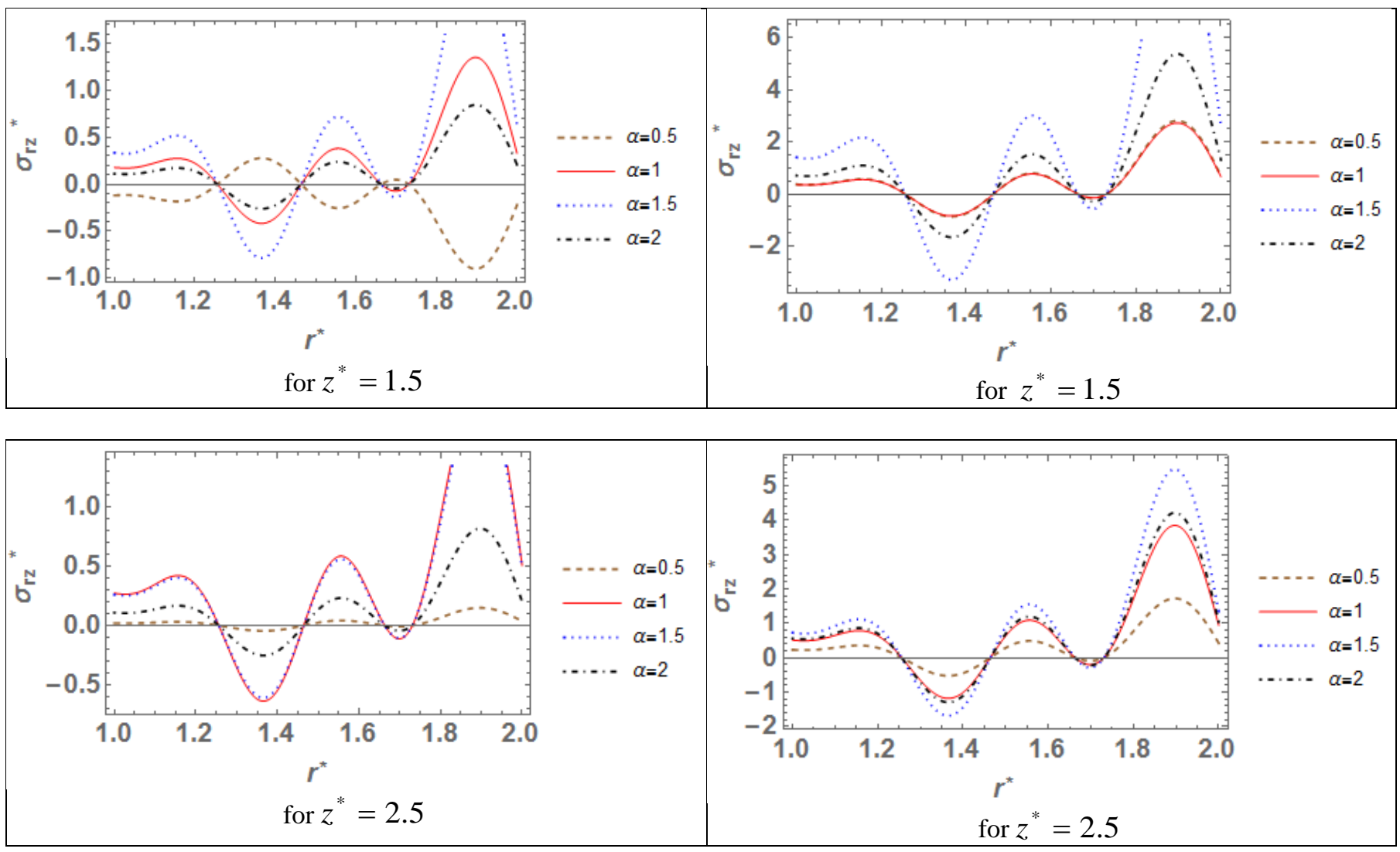

Figure 7. shows dimensionless shear stress distributions

Similarly, Figure 5, 6 and 7 represents the variation of dimensionless tangential stress $\sigma_{\theta \theta}{ }^{*}$, axial stress $\sigma_{z z}{ }^{*}$ and shear stress $\sigma_{r z}{ }^{*}$ respectively in radial direction $r^{*}$ for different values of fractional-order parameter $\alpha=0.5, \alpha=1, \alpha=1.5, \alpha=2$ with different values of dimensionless thickness $z^{*}=0.5, z^{*}=1.5$ and $z^{*}=2.5$. It is observed that on changing values of fractional-order parameter $\alpha$ for different thickness significantly affect the stresses distribution for both homogeneous and non homogeneous cylinder. Hence, both the factors can be an important factor for designing new materials applicable to real life situations.

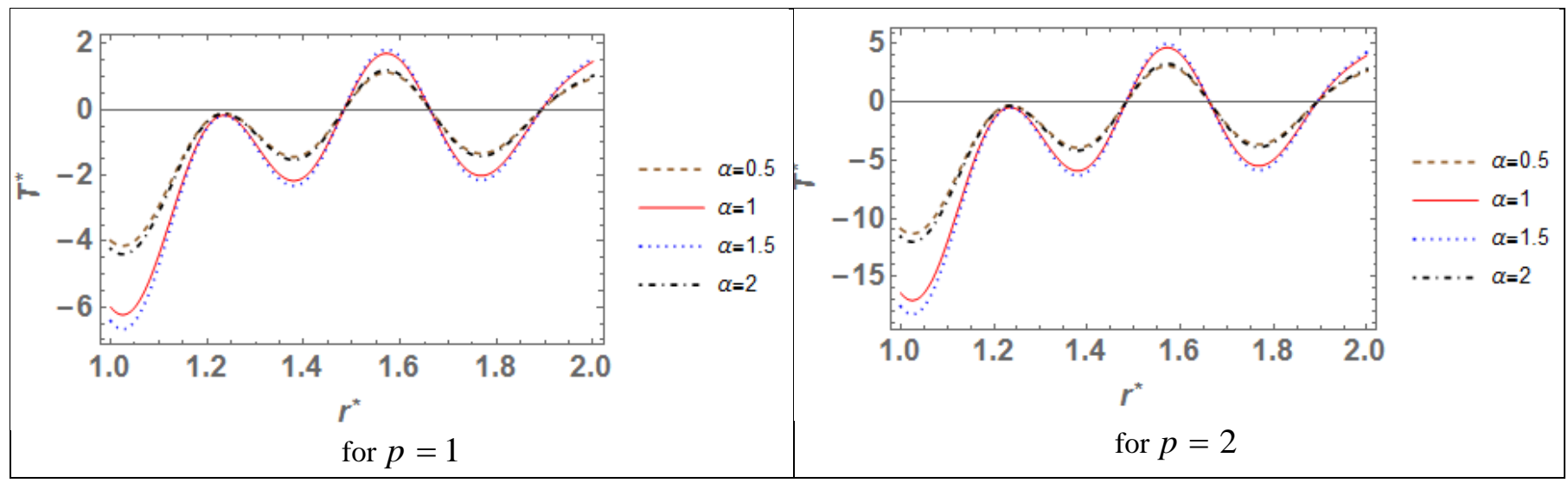




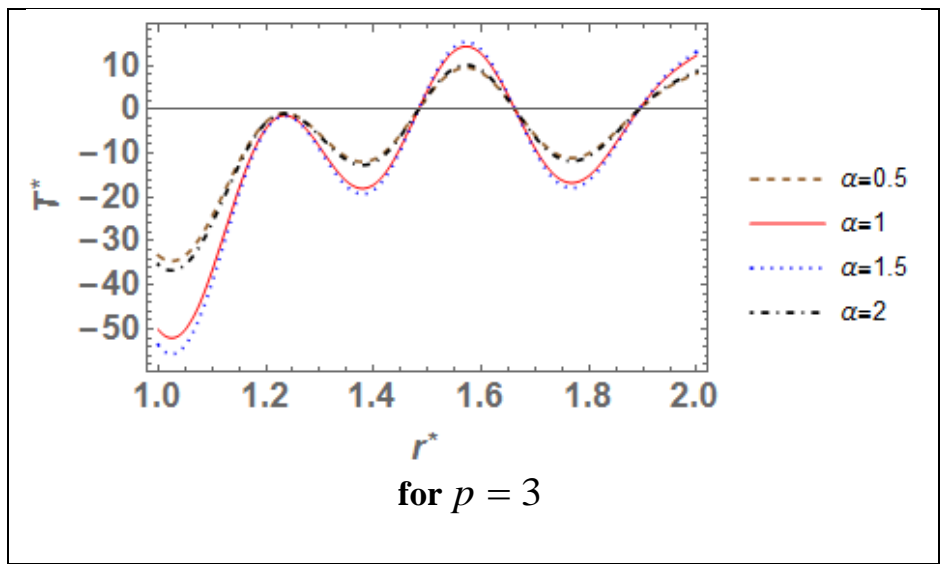

Figure 8. Shows Dimensionless temperature distributions for different inhomogeneity parameter

Figure 8 represents the variation of dimensionless temperature $T^{*}$ in radial direction for different values of fractional-order parameter $\alpha=0.5, \alpha=1, \alpha=1.5, \alpha=2 \quad$ with different values of inhomogeneity parameter $p=1$, $p=2$ and $p=3$. It is seen that the temperature has a finite value at the inner and outer radius because of internal heat generation, which is smoothly increasing in the region $1 \leq r^{*} \leq 1.3$ and then behaves sinusoidal towards the outer radius. It is observed that with increase in the inhomogeneity parameter, the magnitude of temperature is increasing. Also the value of fractional-order parameter $\alpha$ significantly effects the temperature distribution.
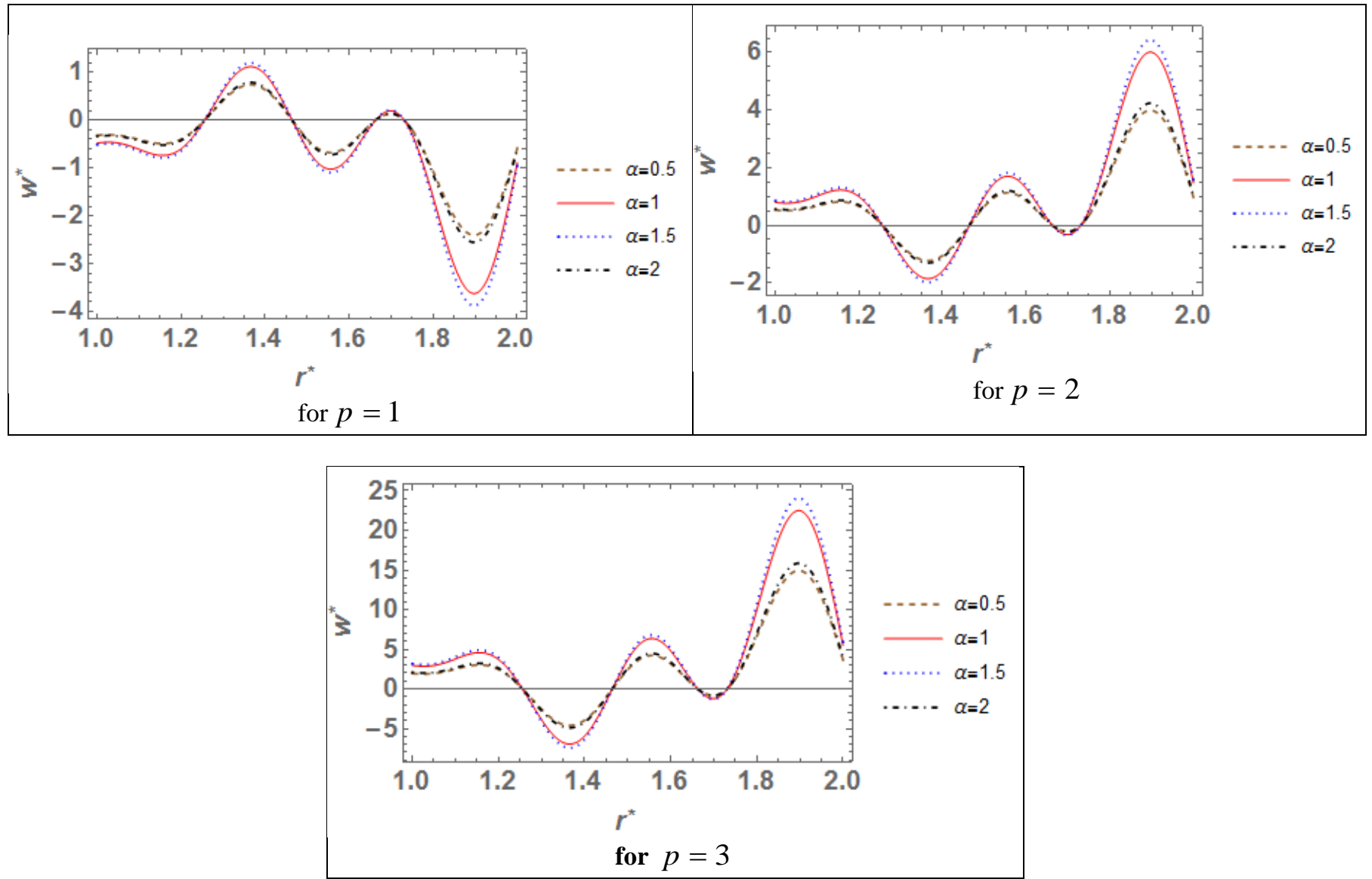
Figure 9 represent variation of dimensionless displacement $w^{*}$ in radial direction $r^{*}$ of hollow cylinder for different inhomogeneity parameter $p$

Figure 9 represent the distribution of dimensionless displacement $w$ in radial direction for different fractional order parameter $\alpha=0.5, \alpha=1$, $p$. It is seen that the displacement distribution is more at the outer radius and also noted that fractional order parameter $\alpha$ directly proportional to displacement.

$\alpha=1.5, \quad \alpha=2$ with inhomogeneity parameter
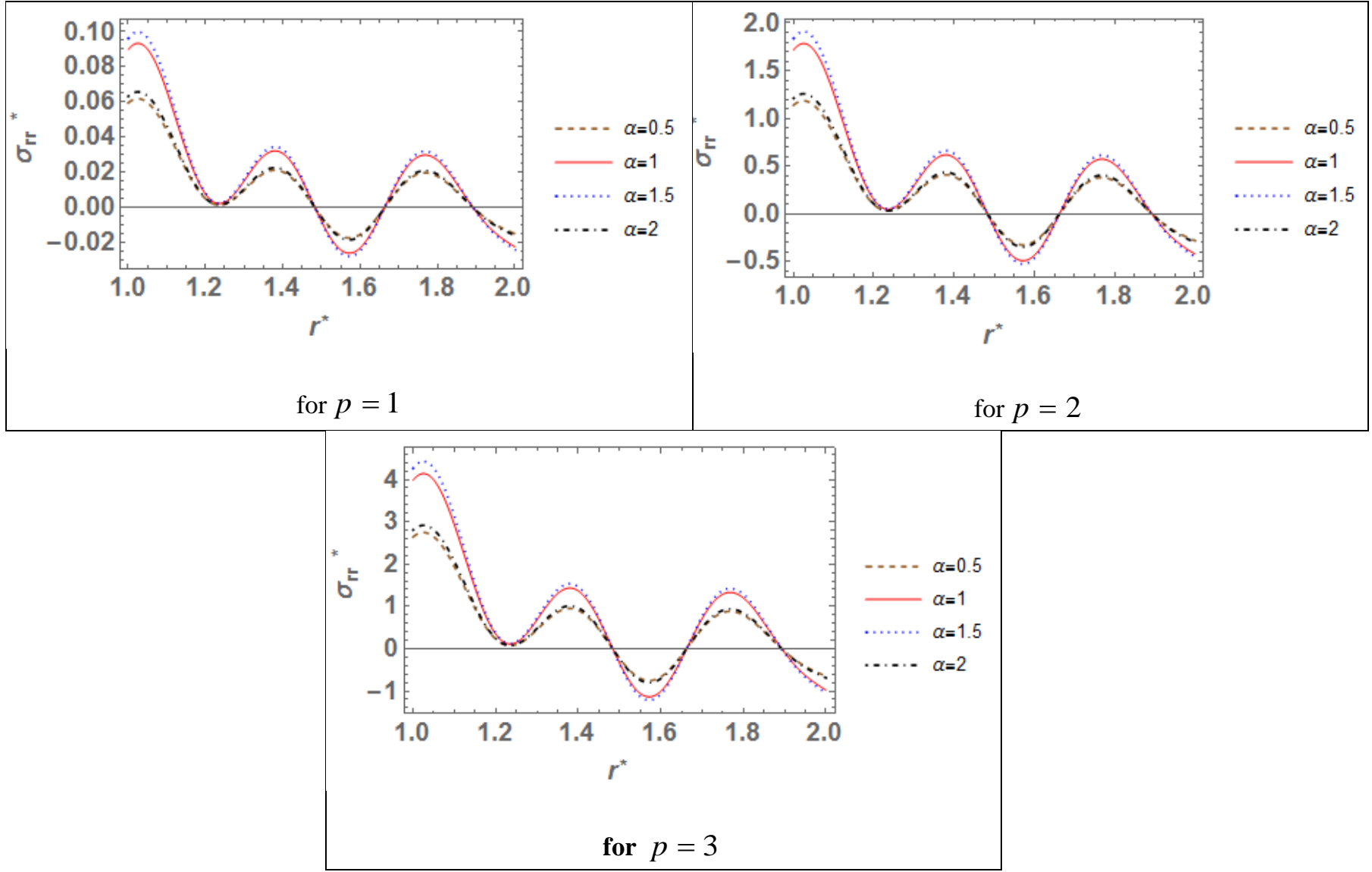

Figure 10 dimensionless radial stress distributions for different inhomogeneity parameter $p$

Figure 10 represent the distribution of dimensionless radial stress $\sigma_{r r}{ }^{*}$ in radial direction $r^{*}$ for different values of $p$ and fractional order parameter $\alpha=0.5, \alpha=1, \alpha=1.5, \alpha=2$. It is seen that initially stresses are more at inner radii and behaves sinusoidal towards the outer radii. Also it is observed that large value of inhomogeneity parameter $p$, the absolute value of radial stress is found decreasing. Also fractional order parameter directly affects the plot. 

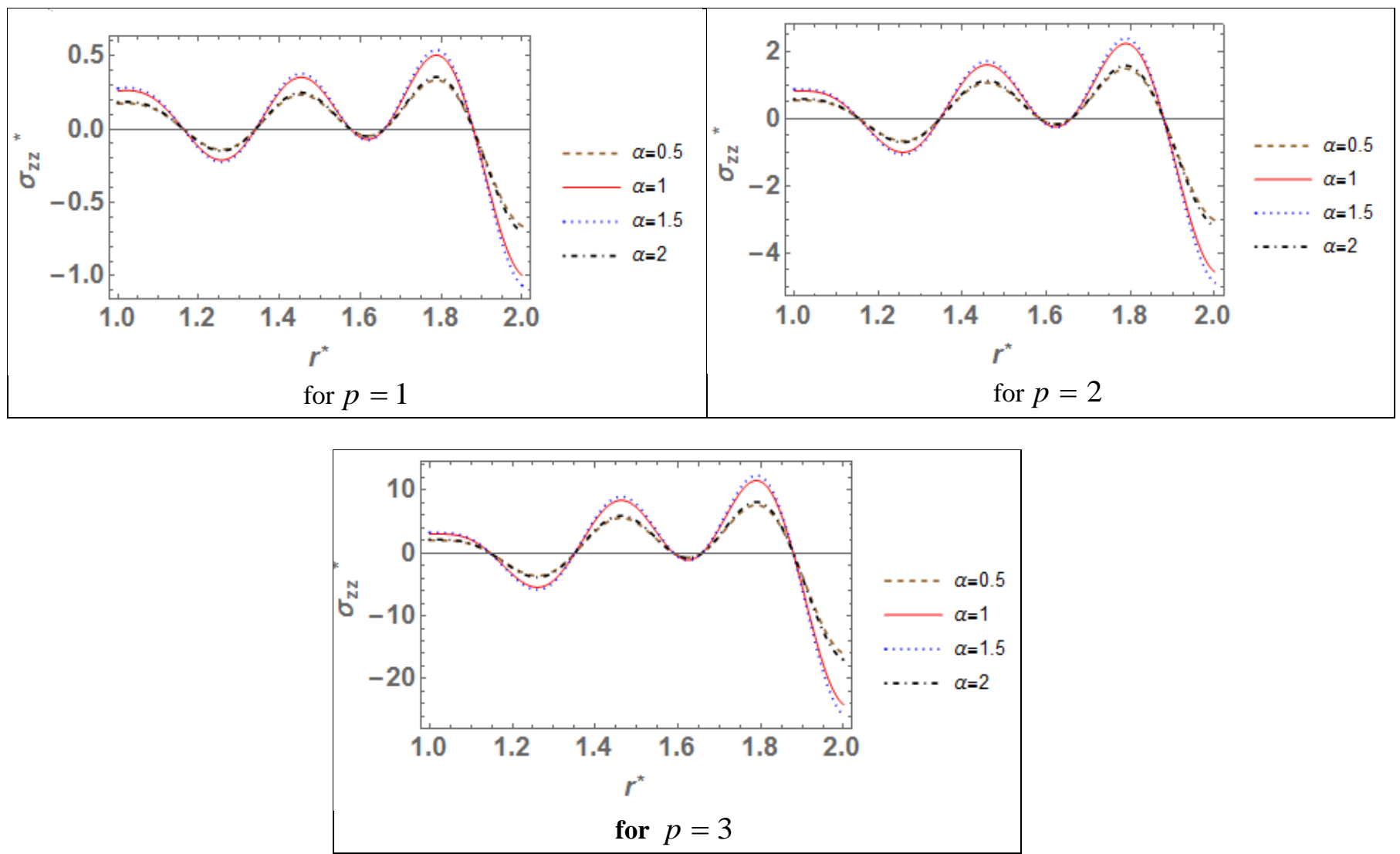

Figure 11 Variation of dimensionless axial stress $\sigma_{z z}{ }^{*}$ in radial direction $r^{*}$ of hollow cylinder for different inhomogeneity parameter $p$

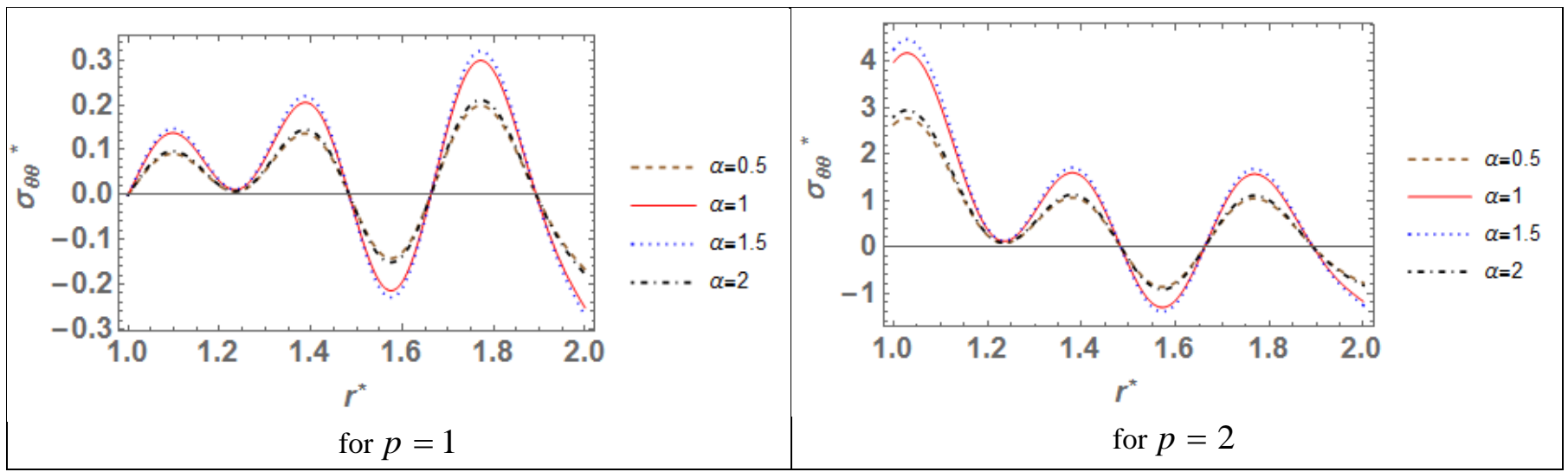




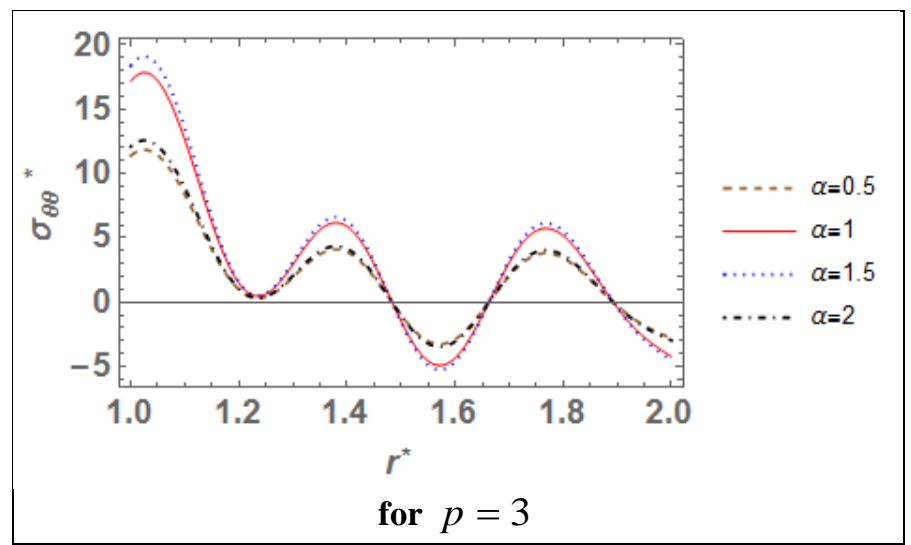

Figure 12 Variation of dimensionless tangential stress ${\sigma_{\theta \theta}}^{*}$ in radial direction $r^{*}$ of hollow for different inhomogeneity parameter $p$
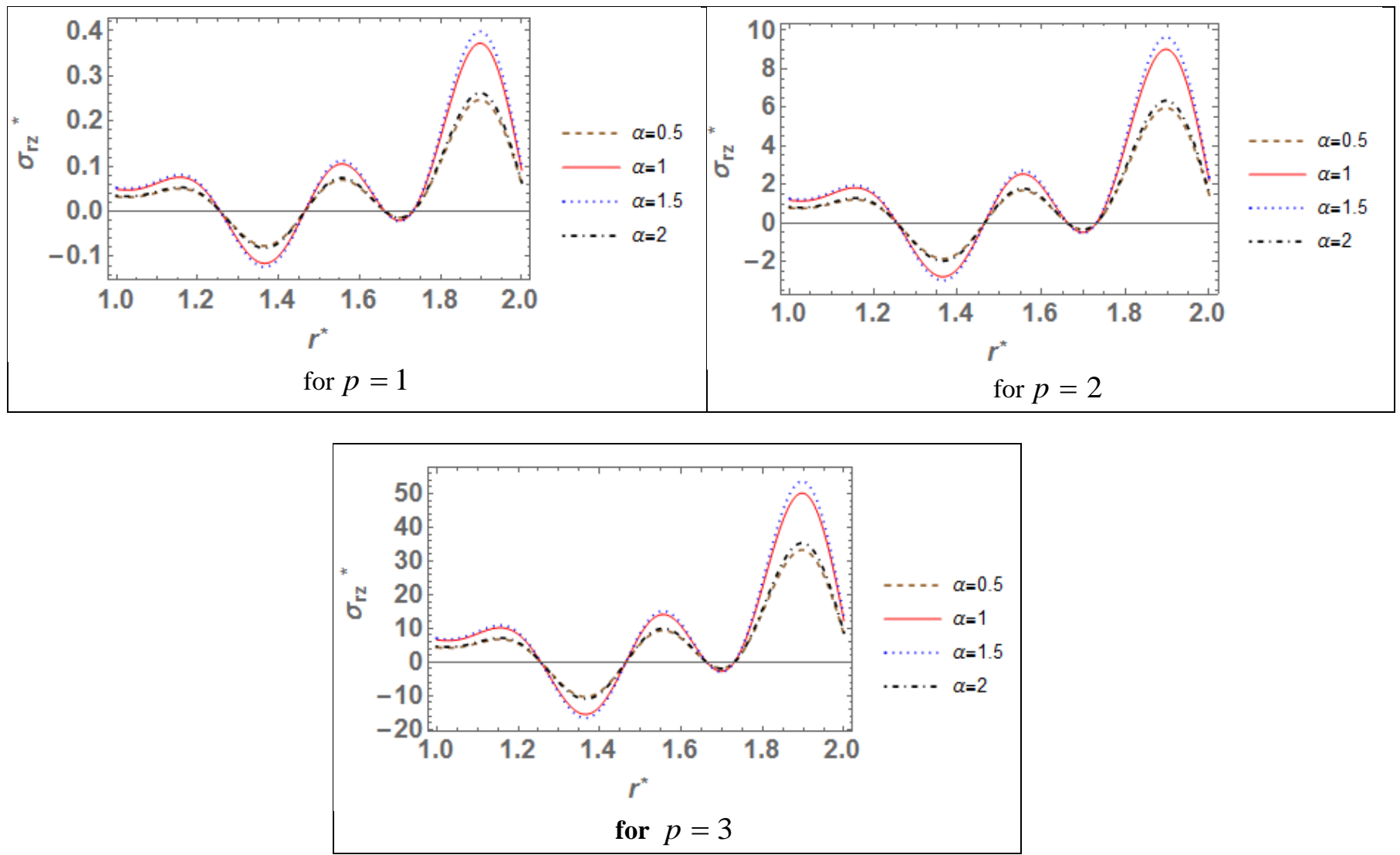

Figure 13 Dimensionless shear stress distributions for different inhomogeneity parameter $p$

Figures 11, 12 and 13 represents the distribution of dimensionless tangential stress, axial stress and shear stress respectively in radial direction for different values of fractional-order parameter $\alpha=0.5, \alpha=1, \alpha=1.5, \alpha=2 \quad$ with different values of inhomogeneity parameter $p=1$, $p=2$ and $p=3$. With increase in the 
inhomogeneity parameter $p$, it is observed that the magnitude of axial stress in less as compared to that of tangential stress and shear stress. Also for different fractional parameter variation in plot is obtained, hence it is concluded that both the parameters play significant role in design of new materials applicable

\section{Conclusion}

In the present paper, we study the time fractional heat conduction equation under zero initial conditions. The integral transform method is used to calculate thermal behaviour with internal heat generation in a thick hollow cylinder subjected to sectional heating on the curved surface. The material properties are assumed to vary by simple power law along axial direction. We solve two-dimensional transient conductivity equation with internal heat generation and obtained its associated thermal stresses for a thick hollow cylinder with inhomogeneous material prop

erties. A mixture of copper and tin metals is chosen for numerical purposes, and the numerical results of transient state temperature field and thermal stresses are examined and illustrated graphically. Investigation for inhomogeneity grading is observed for different value of $p$. From graphical plot it is investigated that (i) temperature, displacement and all stresses is behaved to be sinusoidal along radial direction for different values of dimensionless thickness $z^{*}=0.5$, $z^{*}=1.5$ and $z^{*}=2.5$ (ii) for homogeneous cylinder magnitude of temperature, displacement and

\section{References}

[1] Caputo, M., Linear Model of Dissipation whose Q is almost frequency independent-II, Geophys. J. Royal Astron. Soc., 13, 1967, 529-935.

[2] Lord, H., Shulman, Y., A Generalized Dynamical Theory of Thermoelasticity," J. Mech. Phys. Solids, 15(5), 1967, 299-307.

[3] Caputo, M., Mainardi, F., A new Dissipation model based on Memory Mechanism," Pure Appl. Geophys., 91, 1971, 134-147.

[4] Caputo, M., Mainardi, F., Linear Model of Dissipation in an Elastic Solids, Rivista Del Nuovo Cimento, 1, 1971, 161-198.

[5] Kassir, M. K., Boussinesq Problems for Nonhomogeneous Solid, Journal of the Engineering Mechanics Division, 98(2), 1972, 457-470.

[6] Green, A. E., Lindsay, K. A., Thermoelasticity, J. Elastic., 2(1), 1972, 1-7.

[7] Caputo, M., Vibrations on an infinite viscoelastic Layer with a Dissipative Memory, J. Acoust. Soc. Am., 56, 1974, 897-904.

[8] Edited by the Japan Society of Mechanical Engineers, Elastic Coefficient of Metallic Materials, Japan Society of Mechanical Engineers, 1980. to real life situations. The behaviour of the curves obtained from numerical analysis for the temperature and stress functions shows similar characterization as done in [24]. Also graphical plotting for $(\alpha=1)$ with convective heat exchange boundary conditions found same in previous studies [17].

stresses found high as compared to nonhomogeneous cylinder

(iii) For different values of inhomogeneity parameter $p=1, p=2$ and $p=3$, temperature, displacement and stresses has a finite value at the inner and outer radius due to internal heat generation and then behaves sinusoidal towards the outer radius. (iv) Further fractional order parameter range $0<\alpha<1$ corresponds to weak conductivity and $1<\alpha<2$ corresponds to strong conductivity while $\alpha=1$ corresponds to normal conductivity. (iv) Due to the presence of internal heat generation fluctuations in the temperature distribution and thermoelastic distribution are observed in the neighborhood region of the internal heat generation. Hence we say that nonhomogeneous hollow cylinder with internal heat generation in context of fractional order theory approach predicts lagging response to physical stimulus. Hence, we conclude that above study useful for the design of new materials.

[9] Hata, T., Thermal Stresses in a Nonhomogeneous Thick Plate Under Steady Distribution of Temperature, Journal of Thermal Stresses, 5(1), 1982, $1-11$.

[10] Sugano, Y., Transient Thermal Stresses in a NonHomogeneous Doubly connected region, The Japan Society of Mechanical Engineers, 53, 1987, 941-946.

[11] Sugano, Y., An expression for Transient Thermal Stress in a Nonhomogeneous Plate with Temperature variation through thickness, Ingenieur-Archiv., 57, 1987, 147-156.

[12] Sugano, Y., Transient Thermal Stresses in a Nonhomogeneous Doubly connected region, JSME International Journal Series, 31, 1988. 520-526.

[13] Green, A. E., Naghdi, P. M., Thermoelasticity without energy dissipation, J. Elastic., 31(3), 1993, 189-208.

[14] Hilfer, R., Applications of Fractional Calculus in Physics, World Scientific Publishing, Singapore, 2000.

[15] Awaji, H., Sivakumar, R., Temperature and Stress Distributions in a Hollow Cylinder of Functionally Graded Material: The Case of Temperature- 
Independent Material Properties, Journal of the American Ceramic Society, 84(5), 2001, 1059-1065.

[16] Kim, K. S., Noda, N., Green's Function Approach to Unsteady Thermal Stresses in an Infinite Hollow Cylinder of Functionally Graded Material, Acta Mechanica, 156(3), 2002, 145-161.

[17] Noda, N., Hetnarski, R. B., Tanigawa, Y., Thermal Stresses 2nd Edition, Taylor and Francis, New York, 2003.

[18] Al-Hajri, M., Kalla, S. L., On an Integral Transform involving Bessel Functions, Proceedings of the international conference on Mathematics and its applications, Kuwait, April 5-7, 2004.

[19] Khobragade, N. L., Deshmukh, K. C., Thermal Deformation in a Thin Circular Plate due to a Partially Distributed Heat Supply, Sadhana, 30(4), 2005, 555563.

[20] Ootao, Y., Tanigawa Y., Transient Thermoelastic analysis for a Functionally Graded Hollow Cylinder, Journal of Thermal Stresses, 29, 2005, 1031-1046.

[21] Povstenko, Y. Z., Fractional Heat Conduction Equation and Associated Thermal Stresses, Journal of Thermal Stresses, 28, 2005, 83-102.

[22] Mukhopadhay, S., Kumar, R., A Study of Generalized Thermoelastic interactions in an unbounded medium with a Spherical Cavity, Computers and Mathematics with Applications, 56, 2008, 2329-2339.

[23] Kar, A., Kanoria, M., Generalized Thermoelasticity problem of a Hollow Sphere under Thermal Shock, European Journal of Pure and Applied Mathematics, 2, 2009, 125-146. 2009.

[24] Hosseini, S. M., Akhlaghi, M., Analytical Solution in Transient Thermoelasticity of Functionally Graded Thick Hollow Cylinders, Math. Methods Appl. Sci., 32(15), 2009, 2019-2034.

[25] Ootao, Y., Transient Thermoelastic analysis for a Multilayered Hollow Cylinder with Piecewise Power Law Nonhomogenity, Journal of Solid Mechanics and Materials Engineering, 4, 2010, 1167-1177.

[26] Sherief, H., El-Sayed, A. M. A., Abd El-Latief, A. M., Fractional Order Theory of Thermoelasticity, International Journal of Solids Structure, 47(2), 2010, 269-275.

[27] Povstenko, Y. Z., Fractional Radial Heat Conduction in an infinite medium with a Cylindrical Cavity and associated Thermal Stresses, Mech. Res. Commun., 37, 2010, 436-440.

[28] Ehteram, M. A., Sadighi, M., Tabrizi, H. B., Analytical Solution for Thermal Stresses of Laminated Hollow Cylinders under Transient Nonuniform Thermal Loading, Mechanika, 17(1), 2011, 30-37.

[29] Povstenko, Y. Z., Non-Axisymmetric Solutions to Time-Fractional Diffusion-Wave Equation in an Infinite Cylinder, Fract. Calc. Appl. Anal., 14(3), 2011, 418-435.
[30] Povstenko, Y. Z., Solutions to Time-Fractional Diffusion-Wave Equation in Cylindrical Coordinates, Advances in Differential Equations, Article no. 930297, 2011.

[31] Povstenko, Y. Z., Non-Axisymmetric Solutions to Time-Fractional Heat Conduction Equation in a Half-Space in Cylindrical Coordinates, Math. Methods Phys.-Mech. Fields, 54(1), 2011, 212-219.

[32] Ezzat, M. A., EL-Karamany, A. S., Fractional Order Theory of a perfect conducting Thermoelastic Medium, Can. J. Phys., 89, 2011, 311-318.

[33] Ezzat, M. A., EL-Karamany, A. S., Theory of Fractional Order in Electro - Thermoelasticity, Eur. J. Mech. A/Solids, 30, 2011, 491-500.

[34] Ezzat, M. A., EL-Karamany, A. S., Fractional Order Heat conduction law in MagnetoThermoelasticity involving two Temperatures, $Z$. Angew. Math. Phys., 62(5), 2011, 937-952.

[35] Ezzat, M. A., EL-Karamany, A. S., On Fractional Thermoelasticity, Math. Mech. Solids, 16(3), 2011, 334-346.

[36] Ezzat, M. A., State Space approach to Thermoelectric Fluid with Fractional Order Heat Transfer, Heat Mass Trans., 48, 2012, 71-82.

[37] Ootao, Y., Tanigawa Y., Transient Thermoelastic Analysis for a Functionally Graded Hollow Circular Disk with Piecewise Power Law Nonhomogenity, Journal of Thermal Stresses, 35, 2012, 75-90.

[38] Sherief, H., El-Sayed, A. M., Behiry, S. H., Raslan, W. E., Using Fractional Derivatives to Generalize the Hodgkin-Huxley Model, Fractional Dynamics and Control, 2012, 275-282.

[39] Sur, A., Kanoria, M., Fractional Order twoTemperature Thermoelasticity with wave speed, Acta Mechanica, 223, 2012, 2685-2701.

[40] Ezzat, M. A., EL-Karamany, A. S., Ezzat, S. M., Two-Temperature Theory in MagnetoThermoelasticity with Fractional Order dual-phase-lag Heat Transfer, Nuc. Eng. Des., 252,2012, 267-277.

[41] Ezzat, A. S., EL-Karamany, A. S. Fayik, M. A., Fractional Order Theory in Thermoelastic Solid with three-phase lag Heat Transfer, Arch. Appl. Mech., 82(4),2012, 557-572.

[42] Youssef, H. M., Two-Dimensional Thermal Shock problem of Fractional Order Generalized Thermoelasticity, Acta Mech., 223, 2012, 1219-1231. [43] Sherief, H., Abd El-Latief, A. M., Application of Fractional Order Theory of Thermoelasticity to a 1D problem for a half-space, ZAMM, 2, 2013, 1-7.

[44] Ezzat, M. A., EL-Karamany, A. S., EL-Bary, A. A., Fayik, M. A., Fractional calculus in Onedimensional Isotropic Thermo-viscoelasticity," Comp. Rendus Mecanique, 341, 2013, 553-566.

[45] Tenreiro, J., Alexandra, M., Trujillo, J., Science Metrics on Fractional Calculus Development since 1966, Fract. Calc. Appl. Anal., 16, 2013, 479-500. 
[46] Aksoy, S., Kurşun, A., Çetin, E., Haboğlu, M. R., Stress analysis of Laminated Cylinders subject to the Thermo-Mechanical Loads, International Journal of Mechanical, Aerospace, Industrial, Mechatronic and Manufacturing Engineering, 8(2), 2014, 244-249.

[47] Fu, J., Chen, Z., Qian, L., Hu, K., Transient Thermoelastic analysis of a Solid Cylinder containing a Circumferential Crack using the $\mathrm{C}-\mathrm{V}$ Heat Conduction Model, Journal of Thermal Stresses, 37(11), 2014, 1324-1345.

[48] Jabbari, M., Hashemitaheri, M., Mojahedin, A., Eslami, M. R., Thermal Buckling analysis of Functionally Graded thin Circular Plate made of Saturated Porous Materials, Journal of Thermal Stresses, 37(2), 2014, 202-220.

[49] Sur, A., Kanoria, M., Fractional Order Generalized Thermoelastic Functionally Graded solid with variable material properties, Journal of Solid Mechanics, 6, 2014, 54-69.

[50] Hussain, E. M., Fractional Order Thermoelastic Problem for an Infinitely Long Solid Circular Cylinder, Journal of Thermal Stresses, 38, 2014, 133145.

[51] Raslan, W., Application of Fractional Order Theory of Thermoelasticity to a 1D problem for a Cylindrical Cavity, Arch. Mech, 66, 2014, 257-267.

[52] Povstenko, Y., Fractional Thermoelasticity, New York, Springer, 2015.

[53] Hussain, E. M., Fractional Order Thermoelastic Problem for an infinitely Long Solid Circular Cylinder, Journal of Thermal Stresses, 38, 2015, 133145.

[54] Kedar, G. D., Deshmukh, K. C., Inverse Heat Conduction Problem in a Semi-infinite Hollow Cylinder and its Thermal Deflection by Quasi-static Approach, International Journal of Applied Mathematics and Computation, 6(2), 2015, 15-21.

[55] Raslan, W. E., Application of Fractional Order Theory of Thermoelasticity in a Thick Plate under Axisymmetric Temperature distribution, Journal of Thermal Stresses, 38(7), 2015, 733-743.

[56] Ezzat, M. A., EL-Karamany, A. S., EL-Bary, A. A., On Thermo-viscoelasticity with variable Thermal conductivity and Fractional-Order Heat Transfer, Int. J. Thermophys., 36(7), 1684-1697, 2015.
[57] Ezzat, M. A., EL-Karamany, A. S., EL-Bary, A. A., Thermo-viscoelastic materials with Fractional Relaxation Operators," Appl. Math. Modell., 39, 2015, 7499-7512.

[58] Ezzat, M. A., EL-Bary, A. A., Unified Fractional Derivative Models of Magneto-Thermoviscoelasticity theory, Archives of Mechanics, 68, 2016, 285-308.

[59] Xiong, C., Guo, Y., Effect of variable properties and Moving Heat Source on Magneto Thermoelastic Problem under Fractional Order Thermoelasticity, Advanced in Material Science and Engineering, 2016, $1-12$.

[60] Sherief, H. H., Hamza, F A., Modeling of variable Thermal Conductivity in a Generalized Thermoelastic infinitely Long Hollow Cylinder, Meccanica, 51, 2016, 551-558.

[61] Zhang, X. Y., Li, X. F., Transient Response of a Hygrothermoelastic Cylinder based on Fractional Diffusion Wave Theory, Journal of Thermal Stresses, 40, 2017, 1575-1594.

[62] Zhang, X. Y., Peng Y., Li, X.-F., Time-Fractional Hygrothermoelastic problem for a Sphere Subjected to Heat and Moisture Flux, Journal of Heat Transfer, 140,122002, 2018.

[63] Khobragade, N. L., Kumar, N., Thermal Deflection and Stresses of a Circular Disk Due to Partially Distributed Heat Supply by Application of Fractional Order Theory, Journal of Computer and Mathematical Sciences, 10(3), 2019, 429-437.

[64] Khobragade, N. L., Kumar, N., Study of Thermoelastic Deformation of a Solid Circular Cylinder by Application of Fractional Order Theory, Journal of Computer and Mathematical Sciences, 10(3), 2019, 438-444.

[65] Khobragade, N. L., Lamba, N. K., Magnetothermodynamic Stress Analysis of an Orthotropic Solid Cylinder by Fractional Order Theory Application, Research \& Reviews: Journal of Physics, 8(1), 2019, 37-45.

[66] Khobragade, N. L., Lamba, N. K., Modeling of Thermoelastic Hollow Cylinder by the Application of Fractional Order Theory, Research \& Reviews: Journal of Physics, 8, (1), 2019, 46-57. 